\title{
ANISOTROPY RECONSTRUCTION FROM WAVE FRONTS IN TRANSVERSELY ISOTROPIC ACOUSTIC MEDIA
}

\author{
JOYCE R. MCLAUGHLIN ${ }^{\dagger}$, DANIEL RENZI ${ }^{\dagger}$, AND JEONG-ROCK YOON§
}

\begin{abstract}
This paper considers an inverse problem for a transversely isotropic 3D acoustic medium where there is one preferred direction called the fiber direction along which the wave propagates fastest and there is no preferred wave propagation direction in the isotropic plane, that is the plane orthogonal to the fiber direction. In this medium the parameters to be recovered are: (1) the wave speed for a wave propagating in the direction along the fiber; (2) the wave speed for a wave propagating in any direction which is orthogonal to the fiber direction; and (3) the unit fiber direction itself. So four scalar functions are to be recovered. The data is the positions of four distinct wave fronts as the corresponding waves propagate through the medium. The mathematical relation, that is the Eikonal equation, between the wavefront locations and the four unknown functions is nonlinear. Here it is established, perhaps surprisingly, that corresponding to the given data set, there can be up to four possible solution quadruples. We present and implement an algorithm to compute each of the possible solutions and show our selection criteria to obtain the correct solution. The Eikonal equation, that relates the wave front positions to the unknown functions, is the same as one obtains for the $\mathrm{SH}$ wave which propagates in a linear elastic system.
\end{abstract}

Key words. elastography, inverse problem, arrival time, anisotropic wave equation, transversely isotropic medium, fiber direction.

AMS subject classifications. 35R30, 62P10, 92C55

1. Introduction. Motivated by wave propagation directional dependence in tissue, the goal of this paper is to identify directionally dependent stiffness properties from multiple wave fronts. The wave propagation model is an anisotropic wave equation where the medium has one preferred direction, that we designate as the fiber direction, where it has a faster wave speed and the waves propagating in the plane orthogonal to this preferred direction are slower and exhibit no directional dependence. Our goal is the recovery of the unit fiber direction and the ratio of each of two distinct stiffness coefficients to the density. The square roots of these two ratios define the wave speed in the fiber direction and in the plane orthogonal to the fiber. We show that in 3D there can be up to four discrete solution triples: two wave speeds and the fiber direction, from four distinct wave fronts. The fact that there is a discrete set of solutions is a direct result of the nonlinear relations, governed by Eikonal equation, between wave front directions, wave speeds and the fiber direction.

Shear stiffness recovery has been of interest for about fifteen years where several experiments are being investigated: (1) tissue is compressed as stiff tissue compresses less $[4,17] ;(2)$ single frequency excitation where stiff tissue exhibits low amplitude and stiffness characteristics can be recovered from amplitude variations [8, 15, 19, 20]; (3) crawling or holographic waves are produced with excitations at two nearby frequencies and phase wave speed can be recovered [14, 22]; (4) interior radiation force excitation at a single point produced by a single ultrasound beam [16]; (5) interior radiation force excitation produced with two ultrasound beams whose excitation frequency difference is in the $\mathrm{KHz}$ range [7]; (6) tissue surface line sources, or supersonic imaging which effectively produces line sources orthogonal to the tissue surface, produce propagating

\footnotetext{
${ }^{\dagger}$ Department of Mathematical Sciences, Rensselaer Polytechnic Institute, Troy, NY 12180 (mclauj@rpi.edu, renzid@rpi.edu).

$\S$ Department of Mathematical Sciences, Clemson University, Clemson, SC 29634-0975 (jryoon@clemson.edu).
} 


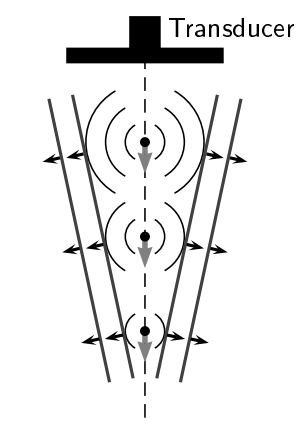

Pulses are made quickly from top to bottom

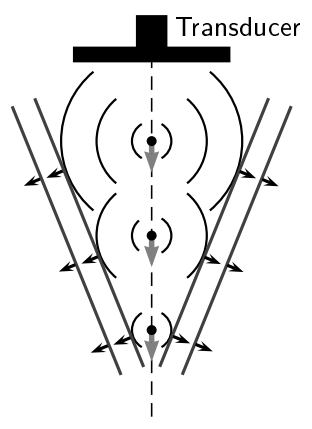

Pulses are made slowly from top to bottom

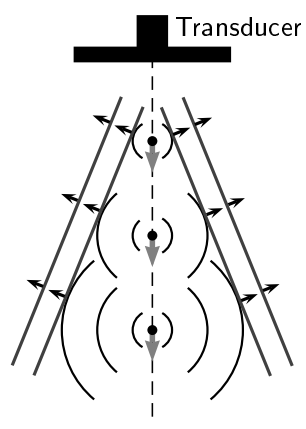

Pulses are made slowly

from bottom to top

FIG. 1.1. Cartoon of three possible conical wave fronts (2D view) produced by a succession of interior radiation force pushes. Transducer focuses ultrasound beams to produce interior radiation force pushes and changes focal depth successively, either from top to bottom or vice versa.

waves with identifiable fronts $[2,3]$; the propagating front locations can be utilized to recover tissue properties. In each of the above six cases the goal is to image either: (a) shear wave speed which is roughly $3 \mathrm{~m} / \mathrm{sec}$ in normal isotropic tissue and can more than double in abnormal tissue; or (b) the shear stiffness which can increase more than four times in abnormal tissue. The aim is to identify abnormal inclusions, which are tumors.

Here we utilize the supersonic imaging experiment. There a line source is approximated by a set of interior radiation force pushes, produced by focused ultrasound beams all at the same frequency, and made successively along a line. This effectively induces a conical wave in $3 \mathrm{D}$ whose angle with the line of the source is determined by how fast the succession of pushes are made and whether or not the pushes begin deep in the tissue and move successively toward the surface or vice versa, see Figure 1.1.

Our aim in this paper is to recover anisotropic tissue properties. Our motivations are: (a) some normal, e.g., muscle, tissue is anisotropic and so mathematical models must include this property; and (b) it has been conjectured, [18, 21], that benign and cancerous tumors may have their own distinguishing anisotropic properties. If indeed the latter conjecture is true, the recovery of anisotropic tumor properties could be of considerable medical importance.

To give some background about what is known in the isotropic case, so as to contrast to the anisotropic case, we recall that previously we have established uniqueness results [10, 11], and the arrival time algorithm [10,12, 13], to reconstruct wave speed in isotropic media. There we show that the positions of one propagating front established the wave speed uniquely; that there is at most one pair, the shear stiffness $\mu$ and the density $\rho$, corresponding to a given single displacement data as a function of space and time, provided the medium is initially at rest. In this paper, we establish that four distinct wave fronts in 3D yield up to four triples: two distinguishing wave speeds and a fiber direction. We note also that given one of the possible triples and the solution of the anisotropic wave equation (as opposed to only the wave front positions) then also there corresponds at most one density $\rho$ corresponding to that triple.

Our paper is organized as follows: In Section 2 we establish that our model has finite propagation speed, that Lipschitz continuous fronts, defined by their arrival 
times, satisfy an anisotropic Eikonal equation, and refer to our very recent result that establishes that arrival times are actually Lipschitz continuous; in Section 3 we give our analysis that there can be up to four discrete solution triples corresponding to four distinct wave fronts; and in Section 4 we show numerical results that includes recoveries of an anisotropic inclusion embedded in an isotropic background.

2. Anisotropic Acoustic Models. We consider anisotropic models where the wave speed represented by $\sqrt{c_{44} / \rho}$ in one preferred direction, which we call the fiber direction, $\vec{f}$, is larger than the wave speed $\sqrt{c_{66} / \rho}$ in the plane orthogonal to the fiber direction. In this plane, which we call the isotropic plane, the wave speed is independent of direction. Our language and notation here are consistent with SHwave propagation in incompressible transversely isotropic linear elastic models which we will consider in a later paper.

Let $\Omega$ be a bounded $\mathcal{C}^{2}$ open connected subset in $\mathbb{R}^{n}$ for $n=2,3$. Assume

$$
\begin{gathered}
\rho \in \mathcal{C}^{0}(\bar{\Omega}), M \in\left[\mathcal{C}^{1}(\bar{\Omega})\right]^{n \times n} \text { is a symmetric matrix function, and } \\
\exists \alpha_{0}>0 \text { such that } \rho(x) \geq \alpha_{0}, \quad \vec{v} \cdot M(x) \vec{v} \geq \alpha_{0}|\vec{v}|^{2}, \quad \forall x \in \bar{\Omega}, \forall \vec{v} \in \mathbb{R}^{n} .
\end{gathered}
$$

Then our anisotropic wave propagation model is

$$
\nabla \cdot(M \nabla u)=\rho u_{t t} \quad \text { in } \Omega \times(0, T)
$$

with homogeneous initial condition, $u(x, 0)=u_{t}(x, 0)=0$ in $\Omega$, and the boundary condition is either Dirichlet or Neumann; $\left.u\right|_{\partial \Omega \times(0, T)}=g$ or $\left.(\nu \cdot M \nabla u)\right|_{\partial \Omega \times(0, T)}=$ $h$, where $\nu$ is the unit outward normal to $\partial \Omega$. This is an anisotropic extension of the frequently used isotropic elastography model, see [1]. We refer the reader to [6] for techniques to establish existence and uniqueness for the initial-boundary value problem associated with $(2.2)$.

Remark 2.1. In terms of the SH-wave motivated assumptions mentioned above, the stiffness matrix $M$ is represented by

$$
M=c_{66} I+\left(c_{44}-c_{66}\right) \vec{f} \otimes \vec{f},
$$

where $|\vec{f}|=1, c_{44}>c_{66}>0$ in $\bar{\Omega}, I$ and $\otimes$ denote the identity matrix and tensor product, respectively. Here our assumption that $c_{44}>c_{66}$ is natural since in biological tissue, e.g., in muscle tissue, the wave speed is fastest in the direction aligned with the fibers [9].

Since our medium is initially at rest, the wave propagates into the medium from the boundary with a propagating front. In our next two theorems, following [10, 11], we establish that the wave whose propagation is governed by the above model has: (1) finite propagation speed; and (2) an arrival time, which we assume to be Lipschitz continuous, that, under this assumption, satisfies the Eikonal equation.

TheOrem 2.2. Assume $\rho$ and $M$ satisfy (2.1). Let $u \in H^{2}(\Omega \times(0, T))$ be a solution of (2.2). Then for any open ball $B_{\epsilon}\left(x_{0}\right) \subset \Omega, u$ has a finite propagation speed in $B_{\epsilon}\left(x_{0}\right) \times(0, T)$ with the maximum speed

$$
c=\sup _{x \in B_{\epsilon}\left(x_{0}\right)} \sqrt{\sigma_{M}(x) / \rho(x)}
$$

where $\sigma_{M}(x)$ is the largest eigenvalue of $M(x)$. 
The proof of the above theorem is along the same line as that in the isotropic case (Theorem 3.4 in [11]), once we redefine the energy by

$$
e(s):=\frac{1}{2} \int_{C_{s}}\left\{\rho\left|u_{t}\right|^{2}+\nabla u \cdot M \nabla u\right\} d x, \quad C_{s}:=B_{\epsilon-c s}\left(x_{0}\right) \times\left\{t=t_{0}+s\right\} .
$$

So we omit the proof.

As in [10] we define the arrival time, $\hat{T}(x)$, of the wave as

$$
\hat{T}(x):=\inf \{t \in(0, T):|u(x, t)|>0\}, \quad x \in \Omega_{u \neq 0},
$$

where $\Omega_{u \neq 0}:=\{x \in \Omega: u(x, t) \neq 0$ for some $t \in(0, T)\}$ and we assume the solution $u$ of (2.2) is continuous. If $\hat{T} \in \mathcal{C}^{1}(\Omega)$, then existing unique continuation results would apply to show that the arrival time, $\hat{T}$, satisfies the Eikonal equation given below. Since our target medium is inhomogeneous, then we expect waves originating at more than one point on the boundary to arrive simultaneously at the same interior points of $\Omega$. In this case $\hat{T}(x)$ could have kinks or at least be non-differentiable there. Hence we assume $\hat{T}(x)$ is Lipschitz continuous and establish the following theorem.

TheOrem 2.3. Assume $\rho \in \mathcal{C}^{1}(\bar{\Omega})$ in addition to $(2.1)$. Let $u \in H^{2}(\Omega \times(0, T)) \cap$ $\mathcal{C}^{0}(\Omega \times(0, T))$ be a solution of $(2.2)$ with $u(x, 0)=u_{t}(x, 0)=0$ in $\Omega$, and one of the boundary condition; $\left.u\right|_{\partial \Omega \times(0, T)}=g$ or $\left.(\nu \cdot M \nabla u)\right|_{\partial \Omega \times(0, T)}=h$. Suppose further that the arrival time $\hat{T}: \Omega_{u \neq 0} \rightarrow[0, T]$ is Lipschitz continuous. Then $\hat{T}$ satisfies the following Eikonal equation

$$
\rho=\nabla \hat{T} \cdot M \nabla \hat{T} \quad \text { a.e. in } \Omega_{u \neq 0} .
$$

In particular, when $M$ is given in the form of (2.3), our Eikonal equation becomes

$$
\frac{1}{|\nabla \hat{T}|^{2}}=\frac{c_{66}}{\rho}+\left(\frac{c_{44}}{\rho}-\frac{c_{66}}{\rho}\right)\left|\frac{\nabla \hat{T}}{|\nabla \hat{T}|} \cdot \vec{f}\right|^{2} .
$$

Proof. Since $\hat{T}$ is Lipschitz continuous, $\nabla \hat{T}$ is well-defined almost everywhere. Note that (2.5) is merely a necessary condition for $t=\hat{T}(x)$ to be a characteristic surface with respect to the hyperbolic equation $\rho u_{t t}=\nabla \cdot(M \nabla u)$. If we suppose that $t=\hat{T}(x)$ is a noncharacteristic surface, we can draw a contradiction as done in Theorem 2.10 in [10], which is based on Theorem 3.6 in [5] and a lemma at page 544 of [6]. See [10] for the details. $\square$

Remark 2.4. In fact, $\hat{T}$ according to the definition (2.4) may be discontinuous even if the solution $u$ is infinitely smooth. However, in this paper we adopt this definition to make arguments simpler and clearer. Modifying the definition of arrival time by

$\hat{T}(x):=\inf \left\{t \in(0, T]:\|u\|_{L^{2}(V \times(0, t))}>0, \forall\right.$ open $V \subset \Omega$ with $\left.x \in V\right\}, \quad x \in \Omega \backslash \Omega_{E}$,

where $\Omega_{E}:=\bigcup\left\{V \subset \Omega\right.$ is an open set satisfying $\left.\|u\|_{L^{2}(V \times(0, T))}=0\right\}$, we have recently established that $\hat{T}: \Omega \backslash \Omega_{E} \rightarrow(0, T]$ is actually Lipschitz continuous. This result will be addressed soon.

Note that in the anisotropic case, the wave does not always propagate in the direction orthogonal to the wave front (group or ray velocity is not always the same as 
phase velocity). Nevertheless under the assumption that $\hat{T}(x)$ is Lipschitz continuous, the phase wave speed, $c(x)$, in the direction orthogonal to the front, satisfies

$$
c(x)|\nabla \hat{T}|=1, \quad c(x)=c(x, \nabla \hat{T}),
$$

and can be determined by the methods given in $[10,12,13]$. In later sections, we will assume that this speed, $c(x)$, has been determined from $\hat{T}$ so when we solve the inverse problem: find $\left(c_{66} / \rho, c_{44} / \rho, \vec{f}\right)$ from multiple arrival times, we will assume we know both $\hat{T}(x)$ and $c(x)$.

Remark 2.5. In later paper we will consider a transversely isotropic elastic medium. Note that, then (2.6) will be the Eikonal equation with $M$ defined as in equation (2.3), satisfied by the $S H$-wave phase $\psi(x)$ in a geometric optics expansion, $\vec{u}=\vec{a} e^{i \omega(t-\psi(x))}$, where $\vec{a}=\vec{a}_{0}+\frac{1}{i \omega} \vec{a}_{1}+\frac{1}{(i \omega)^{2}} \vec{a}_{2}+\cdots$ is an asymptotic series with $\omega \gg 1$.

3. Reconstruction Using Four Measurements. Having established the intrinsically nonlinear Eikonal equations (2.6) and (2.7) in Section 2 we address the utilization of these equations to recover the three unknown quantities $\left(c_{66} / \rho, c_{44} / \rho, \vec{f}\right)$ from wave fronts $\hat{T}$. Since $|\vec{f}|=1$ this means that in $3 \mathrm{D}$ we have four scalar functions to recover. It is natural then to investigate the inverse problem:

$$
\text { find }\left(\frac{c_{66}}{\rho}, \frac{c_{44}}{\rho}, \vec{f}\right) \text { from four distinct wave fronts }\left\{\hat{T}_{j}\right\}_{j=1}^{4} \text {. }
$$

Perhaps surprisingly, our analysis establishes that we can have a finite discrete (up to four) set of triples that correspond to given four distinct propagating wave fronts. We make this statement more precise below.

Let $\left\{\hat{T}_{j}\right\}_{j=1}^{4}$ be four given arrival time data. Define the unit wave normal and the corresponding phase wave speed by $\vec{n}_{j}:=\nabla \hat{T}_{j} /\left|\nabla \hat{T}_{j}\right|$ and $c_{j}:=1 /\left|\nabla T_{j}\right|$, respectively. Recall $c_{j}$ can be estimated by solving (2.7) based on the methods given in $[10,12,13]$. Then the Eikonal equation (2.6) becomes

$$
c_{j}^{2}=\tilde{c}_{66}+\left(\tilde{c}_{44}-\tilde{c}_{66}\right)\left|\vec{f} \cdot \vec{n}_{j}\right|^{2}, \quad j=1,2,3,4,
$$

where we define $\tilde{c}_{66}:=c_{66} / \rho$ and $\tilde{c}_{44}:=c_{44} / \rho$ for convenience. As described in Section 2, we are assuming $\tilde{c}_{44}>\tilde{c}_{66}$, which is a reasonable assumption as the fiber in biological tissue is normally stiffer than the background matrix. Thus $\tilde{c}_{44}$ and $\tilde{c}_{66}$ are the upper and lower bounds of all possible $c_{j}^{2}$, respectively. So we can define $d_{j}:=\sqrt{c_{j}^{2}-\tilde{c}_{66}} \geq 0$ and $\vec{g}:=\sqrt{\tilde{c}_{44}-\tilde{c}_{66}} \vec{f} \neq 0$, and from (3.2) we establish linear relations for $\vec{g}$;

$$
\vec{g} \cdot \vec{n}_{j}= \pm d_{j}, \quad j=1,2,3,4 .
$$

Then our task is to determine $\left(\tilde{c}_{66}, \vec{g}\right)$ from the data $\left\{\left(\vec{n}_{j}, c_{j}\right)\right\}_{j=1}^{4}$. Once we determine $\tilde{c}_{66}$, knowing $\vec{g}$ is equivalent to knowing $\tilde{c}_{44}$ and $\vec{f}$, since $\tilde{c}_{44}=\tilde{c}_{66}+|\vec{g}|^{2}$ and $\vec{f}=\vec{g} /|\vec{g}|$.

In this section, we will show that $\tilde{c}_{66}$ is a root of a fourth order polynomial $p(x)$ (Theorem 3.8), hence we may have four possible $\tilde{c}_{66}$. For each $\tilde{c}_{66}$, we have a generic uniqueness to determine $\vec{g}$ (Corollary 3.15) and an explicit formula for $\vec{g}$ (Theorem 3.9). So we will have at most four possible solutions $\left(\tilde{c}_{66}, \tilde{c}_{44}, \vec{f}\right)$. Since $\tilde{c}_{66}$ can be a multiple root of $p(x)$, despite the generic uniqueness, it may look like we have 
multiple $\tilde{c}_{44}$ and $\vec{f}$ corresponding to a single $\tilde{c}_{66}$ (Theorem 3.10 and 3.12). However, to realize this special case, the data $\left\{\left(\vec{n}_{j}, c_{j}\right)\right\}_{j=1}^{4}$ must satisfy one of a very special set of conditions (3.10)-(3.12) that are unlikely to occur in the actual experiments.

3.1. Coordinate system and data preparation. For convenience, we assume we have a well-prepared data set, defined below, and fix an appropriate coordinate system defined as follows.

Definition 3.1. We define two concepts for our data and a coordinate system.

(a) Data $\left\{\left(\vec{n}_{j}, c_{j}\right)\right\}_{j=1}^{4}$ are called compatible if $c_{1}>c_{2}>c_{3}>c_{4}>0$ and all the followings are not vanishing;

$$
\begin{array}{ll}
\widehat{D}_{1}:=\operatorname{det}\left(\vec{n}_{2}, \vec{n}_{3}, \vec{n}_{4}\right), & \widehat{D}_{2}:=\operatorname{det}\left(\vec{n}_{1}, \vec{n}_{3}, \vec{n}_{4}\right), \\
\widehat{D}_{3}:=\operatorname{det}\left(\vec{n}_{1}, \vec{n}_{2}, \vec{n}_{4}\right), \quad \widehat{D}_{4}:=\operatorname{det}\left(\vec{n}_{1}, \vec{n}_{2}, \vec{n}_{3}\right),
\end{array}
$$

where det denotes the determinant of a matrix consisting of three vectors. This means that at any given point the normals to any three of the four wave fronts are linearly independent.

(b) Data $\left\{\vec{n}_{j}, c_{j}\right\}_{j=1}^{4}$ are called well-prepared if they are compatible and $\vec{n}_{3}, \vec{n}_{4}$ are oriented so that $\widehat{D}_{3}>0$ and $\widehat{D}_{4}>0$.

(c) For convenience, set the coordinate system $\left\{\vec{e}_{1}, \vec{e}_{3}, \vec{e}_{3}\right\}$ utilizing $\vec{n}_{1}$ and $\vec{n}_{2}$ by

$$
\vec{e}_{1}:=\vec{n}_{1}, \quad \vec{e}_{2}:=\frac{\vec{n}_{2}-\left(\vec{n}_{1} \cdot \vec{n}_{2}\right) \vec{n}_{1}}{\left|\vec{n}_{1} \times \vec{n}_{2}\right|}, \quad \vec{e}_{3}:=\frac{\vec{n}_{1} \times \vec{n}_{2}}{\left|\vec{n}_{1} \times \vec{n}_{2}\right|} .
$$

Since $-\vec{n}_{3}$ and $-\vec{n}_{4}$ also satisfy (3.3), any compatible data can be processed into well-prepared data. For well-prepared data, we have

$$
\begin{aligned}
& \vec{n}_{1}=\vec{e}_{1}, \quad \vec{n}_{2}=\left(\vec{n}_{1} \cdot \vec{n}_{2}\right) \vec{e}_{1}+\left|\vec{n}_{1} \times \vec{n}_{2}\right| \vec{e}_{2}, \\
& \vec{n}_{3}=\left(\vec{n}_{1} \cdot \vec{n}_{3}\right) \vec{e}_{1}+\frac{\left(\vec{n}_{1} \times \vec{n}_{2}\right) \cdot\left(\vec{n}_{1} \times \vec{n}_{3}\right) \vec{e}_{2}}{\left|\vec{n}_{1} \times \vec{n}_{2}\right|}+\frac{\widehat{D}_{4} \vec{e}_{3}}{\left|\vec{n}_{1} \times \vec{n}_{2}\right|}=: \alpha_{3} \vec{e}_{1}+\beta_{3} \vec{e}_{2}+\gamma_{3} \vec{e}_{3} \\
& \vec{n}_{4}=\left(\vec{n}_{1} \cdot \vec{n}_{4}\right) \vec{e}_{1}+\frac{\left(\vec{n}_{1} \times \vec{n}_{2}\right) \cdot\left(\vec{n}_{1} \times \vec{n}_{4}\right) \vec{e}_{2}}{\left|\vec{n}_{1} \times \vec{n}_{2}\right|}+\frac{\widehat{D}_{3} \vec{e}_{3}}{\left|\vec{n}_{1} \times \vec{n}_{2}\right|}=: \alpha_{4} \vec{e}_{1}+\beta_{4} \vec{e}_{2}+\gamma_{4} \vec{e}_{3} .
\end{aligned}
$$

Here we have $\gamma_{3}, \gamma_{4}>0$.

3.2. Lemmas based on two or three measurements. Two lemmas using only two or three measurements are presented to show what information can be obtained with the limited data sets.

Lemma 3.2. (Two measurements) Using only two data $\left\{\left(\vec{n}_{j}, c_{j}\right)\right\}_{j=1}^{2}, \tilde{c}_{66}$ can be any arbitrary number in $\left(0, c_{2}^{2}\right]$ and the first two components of $\vec{g}=\vec{g}\left(\tilde{c}_{66}\right)$ are determined up to 4 possibilities in terms of $\tilde{c}_{66}$ and the measured data:

$$
\left(\vec{g} \cdot \vec{e}_{1}, \vec{g} \cdot \vec{e}_{2}\right)= \pm\left(d_{1}, \frac{-d_{2}-d_{1}\left(\vec{n}_{1} \cdot \vec{n}_{2}\right)}{\left|\vec{n}_{1} \times \vec{n}_{2}\right|}\right) \quad \text { or } \quad \pm\left(d_{1}, \frac{d_{2}-d_{1}\left(\vec{n}_{1} \cdot \vec{n}_{2}\right)}{\left|\vec{n}_{1} \times \vec{n}_{2}\right|}\right)
$$

where $d_{1}=\sqrt{c_{1}^{2}-\tilde{c}_{66}}$ and $d_{2}=\sqrt{c_{2}^{2}-\tilde{c}_{66}}$.

Proof. From (3.3) for $j=1,2$ we have $\vec{g} \cdot \vec{e}_{1}=\vec{g} \cdot \vec{n}_{1}= \pm d_{1}$ and

$$
\pm d_{2}=\vec{g} \cdot \vec{n}_{2}=\vec{g} \cdot\left[\left(\vec{n}_{1} \cdot \vec{n}_{2}\right) \vec{e}_{1}+\left|\vec{n}_{1} \times \vec{n}_{2}\right| \vec{e}_{2}\right]=\left(\vec{n}_{1} \cdot \vec{n}_{2}\right)\left(\vec{g} \cdot \vec{e}_{1}\right)+\left|\vec{n}_{1} \times \vec{n}_{2}\right|\left(\vec{g} \cdot \vec{e}_{2}\right) .
$$

Thus we get $\vec{g} \cdot \vec{e}_{2}=\frac{ \pm d_{2}-\left(\vec{n}_{1} \cdot \vec{n}_{2}\right)\left(\vec{g} \cdot \vec{e}_{1}\right)}{\left|\vec{n}_{1} \times \vec{n}_{2}\right|}$, which completes the proof. 
Lemma 3.3. (Three measurements) Using only three data $\left\{\left(\vec{n}_{j}, c_{j}\right)\right\}_{j=1}^{3}, \tilde{c}_{66}$ can be any arbitrary number in $\left(0, c_{3}^{2}\right]$ and $\vec{g}=\vec{g}\left(\tilde{c}_{66}\right)$ is determined up to 4 possibilities in terms of $\tilde{c}_{66}$ and the measured data:

$$
\begin{array}{ll}
\vec{g}_{1}=d_{1} \vec{e}_{1}+\eta \vec{e}_{2}+\frac{1}{\gamma_{3}}\left(d_{3}-\omega\right) \vec{e}_{3}, & \vec{g}_{2}=-d_{1} \vec{e}_{1}-\eta \vec{e}_{2}+\frac{1}{\gamma_{3}}\left(d_{3}+\omega\right) \vec{e}_{3}, \\
\vec{g}_{3}=d_{1} \vec{e}_{1}+\tilde{\eta} \vec{e}_{2}+\frac{1}{\gamma_{3}}\left(d_{3}-\tilde{\omega}\right) \vec{e}_{3}, & \vec{g}_{4}=-d_{1} \vec{e}_{1}-\tilde{\eta} \vec{e}_{2}+\frac{1}{\gamma_{3}}\left(d_{3}+\tilde{\omega}\right) \vec{e}_{3},
\end{array}
$$

where $d_{j}=\sqrt{c_{j}^{2}-\tilde{c}_{66}}$ for $j=1,2,3, \eta=\frac{-d_{2}-d_{1}\left(\vec{n}_{1} \cdot \vec{n}_{2}\right)}{\left|\vec{n}_{1} \times \vec{n}_{2}\right|}, \tilde{\eta}=\frac{d_{2}-d_{1}\left(\vec{n}_{1} \cdot \vec{n}_{2}\right)}{\left|\vec{n}_{1} \times \vec{n}_{2}\right|}$, $\omega=d_{1} \alpha_{3}+\eta \beta_{3}$ and $\tilde{\omega}=d_{1} \alpha_{3}+\tilde{\eta} \beta_{3}$. Note that $\vec{g}_{k} \cdot \vec{n}_{3}=d_{3}>0, k=1,2,3,4$.

Proof. From (3.3) for $j=3$ we get $\pm d_{3}=\vec{g} \cdot \vec{n}_{3}=\alpha_{3}\left(\vec{g} \cdot \vec{e}_{1}\right)+\beta_{3}\left(\vec{g} \cdot \vec{e}_{2}\right)+\gamma_{3}\left(\vec{g} \cdot \vec{e}_{3}\right)$. From Lemma 3.2 we get

$$
\vec{g} \cdot \vec{e}_{3}= \begin{cases}\frac{1}{\gamma_{3}}\left( \pm d_{3}-\alpha_{3} d_{1}-\beta_{3} \eta\right)=\frac{1}{\gamma_{3}}\left( \pm d_{3}-\omega\right) & \text { if }\left(\vec{g} \cdot \overrightarrow{e_{1}}, \vec{g} \cdot \vec{e}_{2}\right)=\left(d_{1}, \eta\right) \\ \frac{1}{\gamma_{3}}\left( \pm d_{3}+\alpha_{3} d_{1}+\beta_{3} \eta\right)=\frac{1}{\gamma_{3}}\left( \pm d_{3}+\omega\right) & \text { if }\left(\vec{g} \cdot \overrightarrow{e_{1}}, \vec{g} \cdot \vec{e}_{2}\right)=-\left(d_{1}, \eta\right) \\ \frac{1}{\gamma_{3}}\left( \pm d_{3}-\alpha_{3} d_{1}-\beta_{3} \tilde{\eta}\right)=\frac{1}{\gamma_{3}}\left( \pm d_{3}-\tilde{\omega}\right) & \text { if }\left(\vec{g} \cdot \overrightarrow{e_{1}}, \vec{g} \cdot \vec{e}_{2}\right)=\left(d_{1}, \tilde{\eta}\right) \\ \frac{1}{\gamma_{3}}\left( \pm d_{3}+\alpha_{3} d_{1}+\beta_{3} \tilde{\eta}\right)=\frac{1}{\gamma_{3}}\left( \pm d_{3}+\tilde{\omega}\right) & \text { if }\left(\vec{g} \cdot \overrightarrow{e_{1}}, \vec{g} \cdot \vec{e}_{2}\right)=-\left(d_{1}, \tilde{\eta}\right)\end{cases}
$$

Thus we have eight possibilities:

$$
\begin{aligned}
\left(\begin{array}{c}
\vec{g} \cdot \vec{e}_{1} \\
\vec{g} \cdot \vec{e}_{2} \\
\vec{g} \cdot \vec{e}_{3}
\end{array}\right)= & \left(\begin{array}{c}
d_{1} \\
\eta \\
\frac{d_{3}-\omega}{\gamma_{3}}
\end{array}\right), \quad\left(\begin{array}{c}
-d_{1} \\
-\eta \\
\frac{d_{3}+\omega}{\gamma_{3}}
\end{array}\right), \quad\left(\begin{array}{c}
d_{1} \\
\tilde{\eta} \\
\frac{d_{3}-\tilde{\omega}}{\gamma_{3}}
\end{array}\right), \quad\left(\begin{array}{c}
-d_{1} \\
-\tilde{\eta} \\
\frac{d_{3}+\tilde{\omega}}{\gamma_{3}}
\end{array}\right), \\
& \left(\begin{array}{c}
d_{1} \\
\eta \\
\frac{-d_{3}-\omega}{\gamma_{3}}
\end{array}\right), \quad\left(\begin{array}{c}
-d_{1} \\
-\eta \\
\frac{-d_{3}+\omega}{\gamma_{3}}
\end{array}\right), \quad\left(\begin{array}{c}
d_{1} \\
\tilde{\eta} \\
\frac{-d_{3}-\tilde{\omega}}{\gamma_{3}}
\end{array}\right), \quad\left(\begin{array}{c}
-d_{1} \\
-\tilde{\eta} \\
\frac{-d_{3}+\tilde{\omega}}{\gamma_{3}}
\end{array}\right) .
\end{aligned}
$$

Since the second line is the same as the first line with opposite sign, which gives the same fiber direction $(\vec{g}$ and $-\vec{g})$ in transversely isotropic media, we select the first line that satisfies $\vec{g} \cdot \vec{n}_{3}=d_{3}>0$, and label the four triples in that line: $\vec{g}_{1}, \vec{g}_{2}, \vec{g}_{3}, \vec{g}_{4}$.

Remark 3.4. Note that $\left(\vec{g}_{1} \cdot \vec{n}_{k}\right)_{k=1}^{3}=\left(d_{1},-d_{2}, d_{3}\right),\left(\vec{g}_{2} \cdot \vec{n}_{k}\right)_{k=1}^{3}=\left(-d_{1}, d_{2}, d_{3}\right)$, $\left(\vec{g}_{3} \cdot \vec{n}_{k}\right)_{k=1}^{3}=\left(d_{1}, d_{2}, d_{3}\right)$ and $\left(\vec{g}_{4} \cdot \vec{n}_{k}\right)_{k=1}^{3}=\left(-d_{1},-d_{2}, d_{3}\right)$.

3.3. Four measurements. In the previous subsection, we showed that from three measurements $\tilde{c}_{66}$ is a continuous parameter that can be anything in $\left(0, c_{3}^{2}\right]$ and our solution $\left(\tilde{c}_{66}, \vec{g}\right)$ can be any of four continuous families

$$
\left\{\left(\tilde{c}_{66}, \vec{g}_{k}\left(\tilde{c}_{66}\right)\right): \tilde{c}_{66} \in\left(0, c_{3}^{2}\right]\right\}_{k=1}^{4} .
$$

But in this subsection we will show that for four measurements the set of possible $\tilde{c}_{66}$ becomes discrete whose maximum number is at most four, and will provide an explicit formula for $\vec{g}$ corresponding to each $\tilde{c}_{66}$.

LeMma 3.5. For $\left\{\vec{g}_{k}\right\}_{k=1}^{4}$ in Lemma 3.3, we get

$$
\begin{array}{ll}
\vec{g}_{1} \cdot \vec{n}_{4}=\frac{d_{1} \widehat{D}_{1}+d_{2} \widehat{D}_{2}+d_{3} \widehat{D}_{3}}{\widehat{D}_{4}}, & \vec{g}_{2} \cdot \vec{n}_{4}=\frac{-d_{1} \widehat{D}_{1}-d_{2} \widehat{D}_{2}+d_{3} \widehat{D}_{3}}{\widehat{D}_{4}}, \\
\vec{g}_{3} \cdot \vec{n}_{4}=\frac{d_{1} \widehat{D}_{1}-d_{2} \widehat{D}_{2}+d_{3} \widehat{D}_{3}}{\widehat{D}_{4}}, & \vec{g}_{4} \cdot \vec{n}_{4}=\frac{-d_{1} \widehat{D}_{1}+d_{2} \widehat{D}_{2}+d_{3} \widehat{D}_{3}}{\widehat{D}_{4}} .
\end{array}
$$


Proof. Because all the others are analogous, we will show only the first case. From Lemma 3.3, we have

$$
\vec{g}_{1} \cdot \vec{n}_{4}=d_{1} \alpha_{4}+\eta \beta_{4}+\frac{d_{3} \gamma_{4}}{\gamma_{3}}-\frac{\omega \gamma_{4}}{\gamma_{3}}=\frac{d_{1} \alpha_{4} \gamma_{3}+\eta \beta_{4} \gamma_{3}-\omega \gamma_{4}}{\gamma_{3}}+\frac{d_{3} \widehat{D}_{3}}{\widehat{D}_{4}} .
$$

Since $d_{1} \vec{e}_{2}-\eta \vec{e}_{1}=\frac{d_{1} \vec{n}_{2}+d_{2} \vec{n}_{1}}{\left|\vec{n}_{1} \times \vec{n}_{2}\right|}=\frac{\gamma_{3}\left(d_{1} \vec{n}_{2}+d_{2} \vec{n}_{1}\right)}{\widehat{D}_{4}}$, we get

$$
\begin{aligned}
\frac{d_{1} \alpha_{4} \gamma_{3}+\eta \beta_{4} \gamma_{3}-\omega \gamma_{4}}{\gamma_{3}} & =\frac{d_{1}\left(\alpha_{4} \gamma_{3}-\alpha_{3} \gamma_{4}\right)-\eta\left(\beta_{3} \gamma_{4}-\beta_{4} \gamma_{3}\right)}{\gamma_{3}} \\
& =\frac{\left(d_{1} \vec{e}_{2}-\eta \vec{e}_{1}\right) \cdot\left(\vec{n}_{3} \times \vec{n}_{4}\right)}{\gamma_{3}}=\frac{d_{1} \widehat{D}_{1}+d_{2} \widehat{D}_{2}}{\widehat{D}_{4}},
\end{aligned}
$$

which completes the proof.

TheOREM 3.6. Let well-prepared data $\left\{\vec{n}_{j}, c_{j}\right\}_{j=1}^{4}$ be given. Then $\tilde{c}_{66} \in\left(0, c_{4}^{2}\right]$ is the first function, $c_{66} / \rho$, in the solution of the inverse problem (3.1) if and only if $\tilde{c}_{66}$ satisfies one of the following eight equations

$$
\pm d_{1} \widehat{D}_{1} \pm d_{2} \widehat{D}_{2}+d_{3} \widehat{D}_{3} \pm d_{4} \widehat{D}_{4}=0, \quad d_{j}:=\sqrt{c_{j}^{2}-\tilde{c}_{66}} \geq 0 .
$$

Proof. If $\tilde{c}_{66} \in\left(0, c_{4}^{2}\right]$ is a solution, then there exist $\vec{g}$ and $\left\{d_{j} \geq 0\right\}_{j=1}^{4}$ that satisfy (3.3). By Lemma 3.3, $\vec{g}$ should be one of $\left\{\vec{g}_{k}\right\}_{k=1}^{4}$ and by Remark 3.4 we have $\left(\vec{g} \cdot \vec{n}_{1}, \vec{g} \cdot \vec{n}_{2}, \vec{g} \cdot \vec{n}_{3}, \vec{g} \cdot \vec{n}_{4}\right)=\left( \pm d_{1}, \pm d_{2}, d_{3}, \pm d_{4}\right)$. Thus we have $A X=0$ where

$$
A:=\left(\begin{array}{cc}
\vec{n}_{1} & \mp d_{1} \\
\vec{n}_{2} & \mp d_{2} \\
\vec{n}_{3} & -d_{3} \\
\vec{n}_{4} & \mp d_{4}
\end{array}\right), \quad X:=\left(\begin{array}{c}
\vec{g} \\
1
\end{array}\right) .
$$

For $X$ to be a nontrivial solution, we get $0=\operatorname{det} A=\mp d_{1} \widehat{D}_{1} \pm d_{2} \widehat{D}_{2}-d_{3} \widehat{D}_{3} \pm d_{4} \widehat{D}_{4}$ which proves the necessity. For sufficiency, from Remark 3.4 we know that all four of the $\vec{g}_{k}$ in Lemma 3.3 already satisfy (3.3) for $j=1,2,3$ for any $\tilde{c}_{66} \in\left(0, c_{3}^{2}\right]$. In addition, if $\tilde{c}_{66}$ satisfies one of (3.4), then $\tilde{c}_{66} \leq c_{4}^{2}$ and one of $\vec{g}_{k} \cdot \vec{n}_{4}$ in Lemma 3.5 satisfies $\vec{g}_{k} \cdot \vec{n}_{4}= \pm d_{4}$. So this particular $\vec{g}_{k}$ and $\tilde{c}_{66}$ satisfies (3.3) for $j=1,2,3,4$. Therefore $\tilde{c}_{66}$ is the first function in a solution of (3.1).

Lemma 3.7. Let $\Pi(a, b, c, d)$ be the following alternating product

$$
\Pi(a, b, c, d):=\prod_{i, j, \ell \in\{0,1\}}\left((-1)^{i} a+(-1)^{j} b+c+(-1)^{\ell} d\right) .
$$

Then we have $\Pi(a, b, c, d)=\left(A_{4}+A_{2}\right)^{2}\left(A_{4}-A_{2}\right)^{2}+4\left(A_{1}-A_{3}\right)\left(A_{1} A_{4}^{2}-A_{3} A_{2}^{2}\right)$, where $A_{1}=a^{2}+b^{2}, A_{2}=a^{2}-b^{2}, A_{3}=c^{2}+d^{2}, A_{4}=c^{2}-d^{2}$.

Proof. It can be easily shown by tedious calculation.

THEOREM 3.8. (Determination of $\left.\tilde{c}_{66}\right) \tilde{c}_{66}$ satisfies one of (3.4) if and only if $\tilde{c}_{66} \in\left(0, c_{4}^{2}\right]$ is a root of $p(x):=\Pi\left(\hat{d}_{1} \widehat{D}_{1}, \hat{d}_{2} \widehat{D}_{2}, \hat{d}_{3} \widehat{D}_{3}, \hat{d}_{4} \widehat{D}_{4}\right)$ where $\hat{d}_{j}=\hat{d}_{j}(x):=$ $\sqrt{c_{j}^{2}-x}$. Here $p(x)$ becomes a fourth order polynomial

$$
p(x)=\left(l_{4}+l_{2}\right)^{2}\left(l_{4}-l_{2}\right)^{2}+4\left(l_{1}-l_{3}\right)\left(l_{1} l_{4}^{2}-l_{3} l_{2}^{2}\right), \quad l_{j}:=l_{j}(x)=a_{j} x-b_{j},
$$


where $a_{1}=\widehat{D}_{1}^{2}+\widehat{D}_{2}^{2}, a_{2}=\widehat{D}_{1}^{2}-\widehat{D}_{2}^{2}, a_{3}=\widehat{D}_{3}^{2}+\widehat{D}_{4}^{2}, a_{4}=\widehat{D}_{3}^{2}-\widehat{D}_{4}^{2}, b_{1}=c_{1}^{2} \widehat{D}_{1}^{2}+c_{2}^{2} \widehat{D}_{2}^{2}$, $b_{2}=c_{1}^{2} \widehat{D}_{1}^{2}-c_{2}^{2} \widehat{D}_{2}^{2}, b_{3}=c_{3}^{2} \widehat{D}_{3}^{2}+c_{4}^{2} \widehat{D}_{4}^{2}$ and $b_{4}=c_{3}^{2} \widehat{D}_{3}^{2}-c_{4}^{2} \widehat{D}_{4}^{2}$.

Proof. By definition, $p(x)=\Pi\left(\hat{d}_{1} \widehat{D}_{1}, \hat{d}_{2} \widehat{D}_{2}, \hat{d}_{3} \widehat{D}_{3}, \hat{d}_{4} \widehat{D}_{4}\right)$ is simply a product of the following eight factors

$$
\pm \hat{d}_{1} \widehat{D}_{1} \pm \hat{d}_{2} \widehat{D}_{2}+\hat{d}_{3} \widehat{D}_{3} \pm \hat{d}_{4} \widehat{D}_{4} .
$$

Since $d_{j}=\hat{d}_{j}\left(\tilde{c}_{66}\right)$, the fact that $\tilde{c}_{66}$ satisfies one of (3.4) is equivalent to finding a root of $p(x)$. Moreover, using Lemma 3.7 with $A_{j}=-l_{j}(x)$, we can easily show that $p(x)$ is the fourth order polynomial given in (3.5).

Since $p(x)$ is a fourth order polynomial, we have at most four possible $\tilde{c}_{66}$, and each $\tilde{c}_{66}$ satisfies at least one of (3.4), or equivalently one of the following four equations:

$$
\begin{aligned}
& \left(d_{1} \widehat{D}_{1}+d_{2} \widehat{D}_{2}+d_{3} \widehat{D}_{3}\right)^{2}=d_{4}^{2} \widehat{D}_{4}^{2}, \\
& \left(d_{1} \widehat{D}_{1}+d_{2} \widehat{D}_{2}-d_{3} \widehat{D}_{3}\right)^{2}=d_{4}^{2} \widehat{D}_{4}^{2}, \\
& \left(d_{1} \widehat{D}_{1}-d_{2} \widehat{D}_{2}+d_{3} \widehat{D}_{3}\right)^{2}=d_{4}^{2} \widehat{D}_{4}^{2}, \\
& \left(d_{1} \widehat{D}_{1}-d_{2} \widehat{D}_{2}-d_{3} \widehat{D}_{3}\right)^{2}=d_{4}^{2} \widehat{D}_{4}^{2}
\end{aligned}
$$

Each equation corresponds to a product of two equations in (3.4). So we obtain the following theorem which provides the corresponding $\vec{g}$ (i.e., $\tilde{c}_{44}$ and $\vec{f}$ ) for each case when $\tilde{c}_{66}$ solves one of the above four equations.

TheOREM 3.9. (Determination of $\vec{g})$ Let $\tilde{c}_{66} \in\left(0, c_{4}^{2}\right]$ be a root of $p(x)$ given in (3.5). Then $\tilde{c}_{66}$ satisfies at least one of (3.6)-(3.9) and for each case the corresponding $\vec{g}$ is determined by

$$
\vec{g}=\left\{\begin{array}{lll}
\vec{g}_{1} & \text { if and only if } & \tilde{c}_{66} \text { satisfies (3.6), } \\
\vec{g}_{2} & \text { if and only if } & \tilde{c}_{66} \text { satisfies (3.7), } \\
\vec{g}_{3} & \text { if and only if } & \tilde{c}_{66} \text { satisfies (3.8), } \\
\vec{g}_{4} & \text { if and only if } & \tilde{c}_{66} \text { satisfies (3.9), }
\end{array}\right.
$$

where $\left\{\vec{g}_{k}\right\}_{k=1}^{4}$ are defined in Lemma 3.3 .

Proof. By Remark 3.4, (3.3) is already satisfied for $j=1,2,3$. For $j=4$, i.e., $\vec{g} \cdot \vec{n}_{4}= \pm d_{4}$, it is easily checked by Lemma 3.5 for each case.

Later we will show that generically only one of (3.6)-(3.9) is satisfied for each $\tilde{c}_{66}$, hence the maximum number of possible solutions $\left(\tilde{c}_{66}, \vec{g}\right)$ will be at most four.

3.4. Multiple $\vec{g}$ for a single $\tilde{c}_{66}$. Throughout the rest of the paper we will assume that the data sets are well-prepared. We define three special types of data allowing multiple $\vec{g}$ corresponding to a single $\tilde{c}_{66}$.

$$
\begin{array}{r}
\left|\widehat{D}_{1}\right|<\left|\widehat{D}_{2}\right|, \quad \widehat{D}_{3}<\widehat{D}_{4}, \quad c_{1}^{2} \widehat{D}_{1}^{2}<c_{2}^{2} \widehat{D}_{2}^{2}, \quad c_{3}^{2} \widehat{D}_{3}<c_{4}^{2} \widehat{D}_{4}^{2}, \\
\quad\left(c_{4}^{2} \widehat{D}_{4}^{2}-c_{3}^{2} \widehat{D}_{3}^{2}\right)\left(\widehat{D}_{2}^{2}-\widehat{D}_{1}^{2}\right)=\left(c_{2}^{2} \widehat{D}_{2}^{2}-c_{1}^{2} \widehat{D}_{1}^{2}\right)\left(\widehat{D}_{4}^{2}-\widehat{D}_{3}^{2}\right), \\
\left|\widehat{D}_{1}\right|<\widehat{D}_{3}, \quad\left|\widehat{D}_{2}\right|<\widehat{D}_{4}, \quad c_{1}^{2} \widehat{D}_{1}^{2}<c_{3}^{2} \widehat{D}_{3}^{2}, \quad c_{2}^{2} \widehat{D}_{2}<c_{4}^{2} \widehat{D}_{4}^{2}, \\
\quad\left(c_{4}^{2} \widehat{D}_{4}^{2}-c_{2}^{2} \widehat{D}_{2}^{2}\right)\left(\widehat{D}_{3}^{2}-\widehat{D}_{1}^{2}\right)=\left(c_{3}^{2} \widehat{D}_{3}^{2}-c_{1}^{2} \widehat{D}_{1}^{2}\right)\left(\widehat{D}_{4}^{2}-\widehat{D}_{2}^{2}\right), \\
\left|\widehat{D}_{1}\right|<\widehat{D}_{4}, \quad\left|\widehat{D}_{2}\right|<\widehat{D}_{3}, \quad c_{1}^{2} \widehat{D}_{1}^{2}<c_{4}^{2} \widehat{D}_{4}^{2}, \quad c_{2}^{2} \widehat{D}_{2}<c_{3}^{2} \widehat{D}_{3}^{2}, \\
\quad\left(c_{3}^{2} \widehat{D}_{3}^{2}-c_{2}^{2} \widehat{D}_{2}^{2}\right)\left(\widehat{D}_{4}^{2}-\widehat{D}_{1}^{2}\right)=\left(c_{4}^{2} \widehat{D}_{4}^{2}-c_{1}^{2} \widehat{D}_{1}^{2}\right)\left(\widehat{D}_{3}^{2}-\widehat{D}_{2}^{2}\right) .
\end{array}
$$


First, note that we should have $\tilde{c}_{66}<c_{4}^{2}$ in order to have more than one $\vec{g}$ : The reason is that if $d_{4}=\sqrt{c_{4}^{2}-\tilde{c}_{66}}=0$, then by Theorem 3.9 at least two of (3.6)(3.9) are satisfied implying that at least one of $d_{1} \widehat{D}_{1}, d_{2} \widehat{D}_{2}, d_{3} \widehat{D}_{3}$ is zero which is a contradiction. In the following theorem we will see each of (3.10)-(3.12) actually enforces $\tilde{c}_{66}<c_{4}^{2}$.

TheOrem 3.10. (Two $\vec{g}$ for a single $\tilde{c}_{66}$ )

(a) Both $\vec{g}_{1}$ and $\vec{g}_{2}$ are solutions $\Leftrightarrow$ The data satisfies (3.10) and $\widehat{D}_{1} \widehat{D}_{2}<0$ $\Leftrightarrow \tilde{c}_{66} \in\left(0, c_{4}^{2}\right)$ and it solves $d_{2} \widehat{D}_{2}=-d_{1} \widehat{D}_{1}$ and $d_{4} \widehat{D}_{4}=d_{3} \widehat{D}_{3}$.

Both $\vec{g}_{3}$ and $\vec{g}_{4}$ are solutions $\Leftrightarrow$ The data satisfies (3.10) and $\widehat{D}_{1} \widehat{D}_{2}>0$ $\Leftrightarrow \tilde{c}_{66} \in\left(0, c_{4}^{2}\right)$ and it solves $d_{2} \widehat{D}_{2}=d_{1} \widehat{D}_{1}$ and $d_{4} \widehat{D}_{4}=d_{3} \widehat{D}_{3}$.

For the above two cases, $\tilde{c}_{66}=\frac{c_{2}^{2} \widehat{D}_{2}^{2}-c_{1}^{2} \widehat{D}_{1}^{2}}{\widehat{D}_{2}^{2}-\widehat{D}_{1}^{2}}=\frac{c_{4}^{2} \widehat{D}_{4}^{2}-c_{3}^{2} \widehat{D}_{3}^{2}}{\widehat{D}_{4}^{2}-\widehat{D}_{3}^{2}} \in\left(0, c_{4}^{2}\right)$.

(b) Both $\vec{g}_{1}$ and $\vec{g}_{3}$ are solutions $\Leftrightarrow$ The data satisfies (3.11) and $\widehat{D}_{1}<0$ $\Leftrightarrow \tilde{c}_{66} \in\left(0, c_{4}^{2}\right)$ and it solves $d_{3} \widehat{D}_{3}=-d_{1} \widehat{D}_{1}$ and $d_{4} \widehat{D}_{4}= \pm d_{2} \widehat{D}_{2}$.

Both $\vec{g}_{2}$ and $\vec{g}_{4}$ are solutions $\Leftrightarrow$ The data satisfies (3.11) and $\widehat{D}_{1}>0$ $\Leftrightarrow \tilde{c}_{66} \in\left(0, c_{4}^{2}\right)$ and it solves $d_{3} \widehat{D}_{3}=d_{1} \widehat{D}_{1}$ and $d_{4} \widehat{D}_{4}= \pm d_{2} \widehat{D}_{2}$.

For the above two cases, $\tilde{c}_{66}=\frac{c_{3}^{2} \widehat{D}_{3}^{2}-c_{1}^{2} \widehat{D}_{1}^{2}}{\widehat{D}_{3}^{2}-\widehat{D}_{1}^{2}}=\frac{c_{4}^{2} \widehat{D}_{4}^{2}-c_{2}^{2} \widehat{D}_{2}^{2}}{\widehat{D}_{4}^{2}-\widehat{D}_{2}^{2}} \in\left(0, c_{4}^{2}\right)$.

(c) Both $\vec{g}_{1}$ and $\vec{g}_{4}$ are solutions $\Leftrightarrow$ The data satisfies (3.12) and $\widehat{D}_{2}<0$ $\Leftrightarrow \tilde{c}_{66} \in\left(0, c_{4}^{2}\right)$ and it solves $d_{3} \widehat{D}_{3}=-d_{2} \widehat{D}_{2}$ and $d_{4} \widehat{D}_{4}= \pm d_{1} \widehat{D}_{1}$.

Both $\vec{g}_{2}$ and $\vec{g}_{3}$ are solutions $\Leftrightarrow$ The data satisfies (3.12) and $\widehat{D}_{2}>0$ $\Leftrightarrow \tilde{c}_{66} \in\left(0, c_{4}^{2}\right)$ and it solves $d_{3} \widehat{D}_{3}=d_{2} \widehat{D}_{2}$ and $d_{4} \widehat{D}_{4}= \pm d_{1} \widehat{D}_{1}$.

For the above two cases, $\tilde{c}_{66}=\frac{c_{4}^{2} \widehat{D}_{4}^{2}-c_{1}^{2} \widehat{D}_{1}^{2}}{\widehat{D}_{4}^{2}-\widehat{D}_{1}^{2}}=\frac{c_{3}^{2} \widehat{D}_{3}^{2}-c_{2}^{2} \widehat{D}_{2}^{2}}{\widehat{D}_{3}^{2}-\widehat{D}_{2}^{2}} \in\left(0, c_{4}^{2}\right)$.

Proof. Because all the others are analogous, we will only show (c). For $\vec{g}_{1}$ and $\vec{g}_{4}$ to be the solutions, from Theorem $3.9 \tilde{c}_{66}$ satisfies (3.6) and (3.9). Since $d_{1} \widehat{D}_{1} \neq 0$, we must have $d_{3} \widehat{D}_{3}=-d_{2} \widehat{D}_{2}$, and so $d_{4} \widehat{D}_{4}= \pm d_{1} \widehat{D}_{1}$. For $\vec{g}_{2}$ and $\vec{g}_{3}$ to be the solutions, from Theorem $3.9 \tilde{c}_{66}$ satisfies (3.7) and (3.8). Since $d_{1} \widehat{D}_{1} \neq 0$, we must have $d_{3} \widehat{D}_{3}=d_{2} \widehat{D}_{2}$, and so $d_{4} \widehat{D}_{4}= \pm d_{1} \widehat{D}_{1}$.

Now we will show that the data satisfies (3.12) and $\widehat{D}_{2} \lessgtr 0$, respectively, if $\tilde{c}_{66} \in\left(0, c_{4}^{2}\right)$ solves $d_{3} \widehat{D}_{3}=\mp d_{2} \widehat{D}_{2}$ and $d_{4} \widehat{D}_{4}= \pm d_{1} \widehat{D}_{1}$. First note that $\widehat{D}_{2} \lessgtr 0$, respectively, since $d_{2}, d_{3}>0$. For both cases, we get

$$
\begin{aligned}
& \widehat{D}_{3}^{2}\left(c_{3}^{2}-\tilde{c}_{66}\right)=d_{3}^{2} \widehat{D}_{3}^{2}=d_{2}^{2} \widehat{D}_{2}^{2}=\widehat{D}_{2}^{2}\left(c_{2}^{2}-\tilde{c}_{66}\right), \\
& \widehat{D}_{4}^{2}\left(c_{4}^{2}-\tilde{c}_{66}\right)=d_{4}^{2} \widehat{D}_{4}^{2}=d_{1}^{2} \widehat{D}_{1}^{2}=\widehat{D}_{1}^{2}\left(c_{1}^{2}-\tilde{c}_{66}\right),
\end{aligned}
$$

thus we should have $\tilde{c}_{66}=\frac{c_{3}^{2} \widehat{D}_{3}^{2}-c_{2}^{2} \widehat{D}_{2}^{2}}{\widehat{D}_{3}^{2}-\widehat{D}_{2}^{2}}=\frac{c_{4}^{2} \widehat{D}_{4}^{2}-c_{1}^{2} \widehat{D}_{1}^{2}}{\widehat{D}_{4}^{2}-\widehat{D}_{1}^{2}}$. So we have

$$
\left(c_{3}^{2} \widehat{D}_{3}^{2}-c_{2}^{2} \widehat{D}_{2}^{2}\right)\left(\widehat{D}_{4}^{2}-\widehat{D}_{1}^{2}\right)=\left(c_{4}^{2} \widehat{D}_{4}^{2}-c_{1}^{2} \widehat{D}_{1}^{2}\right)\left(\widehat{D}_{3}^{2}-\widehat{D}_{2}^{2}\right) .
$$

Moreover, since $d_{1}>d_{2}>d_{3}>d_{4}$, we get $\left|\widehat{D}_{1}\right|<\widehat{D}_{4}$ and $\left|\widehat{D}_{2}\right|<\widehat{D}_{3}$. From $\tilde{c}_{66}>0$, we also get $c_{1}^{2} \widehat{D}_{1}<c_{4}^{2} \widehat{D}_{4}^{2}$ and $c_{2}^{2} \widehat{D}_{2}^{2}<c_{3}^{2} \widehat{D}_{3}^{2}$.

Finally we will show $\tilde{c}_{66} \in\left(0, c_{4}^{2}\right)$ and it solves $d_{3} \widehat{D}_{3}=\mp d_{2} \widehat{D}_{2}, d_{4} \widehat{D}_{4}= \pm d_{1} \widehat{D}_{1}$, if the data satisfies (3.12) and $\widehat{D}_{2} \lessgtr 0$, respectively. Set $\tilde{c}_{66}:=\frac{c_{3}^{2} \widehat{D}_{3}^{2}-c_{2}^{2} \widehat{D}_{2}^{2}}{\widehat{D}_{3}^{2}-\widehat{D}_{2}^{2}}=$ $\frac{c_{4}^{2} \widehat{D}_{4}^{2}-c_{1}^{2} \widehat{D}_{1}^{2}}{\widehat{D}_{4}^{2}-\widehat{D}_{1}^{2}}>0$. Since $c_{1}^{2}>c_{4}^{2}$, we get $\tilde{c}_{66}=\frac{c_{4}^{2} \widehat{D}_{4}^{2}-c_{1}^{2} \widehat{D}_{1}^{2}}{\widehat{D}_{4}^{2}-\widehat{D}_{1}^{2}}<\frac{c_{4}^{2} \widehat{D}_{4}^{2}-c_{4}^{2} \widehat{D}_{1}^{2}}{\widehat{D}_{4}^{2}-\widehat{D}_{1}^{2}}=c_{4}^{2}$. Thus 
$\tilde{c}_{66} \in\left(0, c_{4}^{2}\right)$ and solves $d_{3}^{2} \widehat{D}_{3}^{2}=d_{2}^{2} \widehat{D}_{2}^{2}$ and $d_{4}^{2} \widehat{D}_{4}^{2}=d_{1}^{2} \widehat{D}_{1}^{2}$. Since $\widehat{D}_{2} \lessgtr 0, \tilde{c}_{66}$ solves $d_{3} \widehat{D}_{3}=\mp d_{2} \widehat{D}_{2}$, respectively and $d_{4}^{2} \widehat{D}_{4}^{2}=d_{1}^{2} \widehat{D}_{1}^{2}$.

In each case in (3.10)-(3.12), the fourth order polynomial $p(x)$ for $\tilde{c}_{66}$ in Theorem 3.8 is now further simplified. We will use the following theorem to show the generic uniqueness of $\vec{g}$ in Corollary 3.15.

THEOREM 3.11. In each case in (3.10)-(3.12), $p(x)$ in Theorem 3.8 becomes

$$
p(x)= \begin{cases}\left(\left(\widehat{D}_{2}^{2}-\widehat{D}_{1}^{2}\right) x-\left(c_{2}^{2} \widehat{D}_{2}^{2}-c_{1}^{2} \widehat{D}_{1}^{2}\right)\right)^{2} q_{1}(x) & \text { if the data satisfies (3.10), } \\ \left(\left(\widehat{D}_{3}^{2}-\widehat{D}_{1}^{2}\right) x-\left(c_{3}^{2} \widehat{D}_{3}^{2}-c_{1}^{2} \widehat{D}_{1}^{2}\right)\right)^{2} q_{2}(x) & \text { if the data satisfies (3.11), } \\ \left(\left(\widehat{D}_{4}^{2}-\widehat{D}_{1}^{2}\right) x-\left(c_{4}^{2} \widehat{D}_{4}^{2}-c_{1}^{2} \widehat{D}_{1}^{2}\right)\right)^{2} q_{3}(x) & \text { if the data satisfies (3.12), }\end{cases}
$$

where $q_{1}, q_{2}$ and $q_{3}$ are second order polynomials.

Proof. Because all the others are analogous, we will show only the case (3.11). First note that

$$
\begin{gathered}
\widehat{D}_{1}^{2}=\frac{a_{1}+a_{2}}{2}, \quad \widehat{D}_{2}^{2}=\frac{a_{1}-a_{2}}{2}, \quad \widehat{D}_{3}^{2}=\frac{a_{3}+a_{4}}{2}, \quad \widehat{D}_{4}^{2}=\frac{a_{3}-a_{4}}{2}, \\
c_{1}^{2} \widehat{D}_{1}^{2}=\frac{b_{1}+b_{2}}{2}, \quad c_{2}^{2} \widehat{D}_{2}^{2}=\frac{b_{1}-b_{2}}{2}, \quad c_{3}^{2} \widehat{D}_{3}^{2}=\frac{b_{3}+b_{4}}{2}, \quad c_{4}^{2} \widehat{D}_{4}^{2}=\frac{b_{3}-b_{4}}{2},
\end{gathered}
$$

and define

$A:=\frac{a_{4}+a_{3}-a_{2}-a_{1}}{2}=\widehat{D}_{3}^{2}-\widehat{D}_{1}^{2}>0, \quad B:=\frac{b_{4}+b_{3}-b_{2}-b_{1}}{2}=c_{3}^{2} \widehat{D}_{3}^{2}-c_{1}^{2} \widehat{D}_{1}^{2}>0$.

Since we can show $\left(a_{4}-a_{2}\right)\left(b_{3}-b_{1}\right)=\left(a_{3}-a_{1}\right)\left(b_{4}-b_{2}\right)$ from (3.11), we also get $A\left(b_{3}-b_{1}\right)=B\left(a_{3}-a_{1}\right)$ and $A\left(b_{4}-b_{2}\right)=B\left(a_{4}-a_{2}\right)$. Thus we get

$$
A\left(l_{1}(x)-l_{3}(x)\right)=\left(a_{1}-a_{3}\right)(A x-B), \quad A\left(l_{2}(x)-l_{4}(x)\right)=\left(a_{2}-a_{4}\right)(A x-B) .
$$

Hence the polynomial in (3.5) becomes

$$
p(x)=C_{2}^{2}(A x-B)^{2}\left(l_{4}+l_{2}\right)^{2}-4 C_{1}(A x-B) Q(x),
$$

where $C_{1}=\frac{a_{3}-a_{1}}{A}, C_{2}=\frac{a_{4}-a_{2}}{A}$ and

$$
\begin{aligned}
Q(x) & =l_{1} l_{4}^{2}-l_{3}\left[l_{4}-C_{2}(A x-B)\right]^{2} \\
& =\left(l_{1}-l_{3}\right) l_{4}^{2}-l_{3}(A x-B)\left[-2 l_{4} C_{2}+C_{2}^{2}(A x-B)\right] \\
& =(A x-B)\left[2 C_{2} l_{3} l_{4}-C_{1} l_{4}^{2}-C_{2}^{2} l_{3}(A x-B)\right] \\
& =(A x-B)\left[2 C_{2} l_{3} l_{4}-C_{1} l_{4}^{2}+C_{2} l_{3}\left(l_{2}-l_{4}\right)\right]=(A x-B)\left[C_{2} l_{3}\left(l_{4}+l_{2}\right)-C_{1} l_{4}^{2}\right] .
\end{aligned}
$$

Hence we get $p(x)=(A x-B)^{2} q_{2}(x)$ where $q_{2}$ is a second order polynomial given by $q_{2}(x)=C_{2}^{2}\left(l_{4}+l_{2}\right)^{2}+4 C_{1}^{2} l_{4}^{2}-4 C_{1} C_{2} l_{3}\left(l_{2}+l_{4}\right)$.

For three possible $\vec{g}$ corresponding to a single $\tilde{c}_{66}$, we consider another special type of data that satisfies

$$
\left|\widehat{D}_{1}\right|<\left|\widehat{D}_{2}\right|<\widehat{D}_{3}<\widehat{D}_{4}, \quad c_{1}^{2} \widehat{D}_{1}^{2}<c_{2}^{2} \widehat{D}_{2}^{2}<c_{3}^{2} \widehat{D}_{3}<c_{4}^{2} \widehat{D}_{4}^{2},
$$

and there exists a single $K:=\frac{c_{j}^{2} \widehat{D}_{j}^{2}-c_{i}^{2} \widehat{D}_{i}^{2}}{\widehat{D}_{j}^{2}-\widehat{D}_{i}^{2}} \in\left(0, c_{4}^{2}\right)$ for all $j>i$. 
Note that (3.13) implies $\left\{\left(x_{j}, y_{j}\right)=\left(\widehat{D}_{j}^{2}, c_{j}^{2} \widehat{D}_{j}^{2}\right)\right\}$ are on a single straight line with slope $K>0$. From Theorem 3.10 , we can easily prove the following theorem showing exactly when we shall get three $\vec{g}$ corresponding to a single $\tilde{c}_{66}$.

THEOREM 3.12. (Three $\vec{g}$ for a single $\tilde{c}_{66}$ )

(a) $\vec{g}_{1}, \vec{g}_{2}, \vec{g}_{3}$ are solutions $\Leftrightarrow$ The data satisfies (3.13), $\widehat{D}_{1}<0$ and $\widehat{D}_{2}>0$ $\Leftrightarrow \tilde{c}_{66} \in\left(0, c_{4}^{2}\right)$ and it solves $d_{4} \widehat{D}_{4}=d_{3} \widehat{D}_{3}=d_{2} \widehat{D}_{2}=-d_{1} \widehat{D}_{1}$.

(b) $\vec{g}_{1}, \vec{g}_{2}, \vec{g}_{4}$ are solutions $\Leftrightarrow$ The data satisfies (3.13), $\widehat{D}_{1}>0$ and $\widehat{D}_{2}<0$ $\Leftrightarrow \tilde{c}_{66} \in\left(0, c_{4}^{2}\right)$ and it solves $d_{4} \widehat{D}_{4}=d_{3} \widehat{D}_{3}=-d_{2} \widehat{D}_{2}=d_{1} \widehat{D}_{1}$.

(c) $\vec{g}_{1}, \vec{g}_{3}, \vec{g}_{4}$ are solutions $\Leftrightarrow$ The data satisfies (3.13), $\widehat{D}_{1}<0$ and $\widehat{D}_{2}<0$ $\Leftrightarrow \tilde{c}_{66} \in\left(0, c_{4}^{2}\right)$ and it solves $d_{4} \widehat{D}_{4}=d_{3} \widehat{D}_{3}=-d_{2} \widehat{D}_{2}=-d_{1} \widehat{D}_{1}$.

(d) $\vec{g}_{2}, \vec{g}_{3}, \vec{g}_{4}$ are solutions $\Leftrightarrow$ The data satisfies (3.13), $\widehat{D}_{1}>0$ and $\widehat{D}_{2}>0$ $\Leftrightarrow \tilde{c}_{66} \in\left(0, c_{4}^{2}\right)$ and it solves $d_{4} \widehat{D}_{4}=d_{3} \widehat{D}_{3}=d_{2} \widehat{D}_{2}=d_{1} \widehat{D}_{1}$.

In each case, we should have $\tilde{c}_{66}=K \in\left(0, c_{4}^{2}\right)$ given in (3.13).

As in Theorem 3.11, the fourth order polynomial $p(x)$ for $\tilde{c}_{66}$ in Theorem 3.8 is also further simplified when the data satisfies (3.13). The following theorem will also be used to show the generic uniqueness of $\vec{g}$ in Corollary 3.15.

TheOREM 3.13. If the data satisfies (3.13), $p(x)$ in Theorem 3.8 becomes

$$
p(x)=(x-K)^{3}\left(\Pi\left(\widehat{D}_{1}, \widehat{D}_{2}, \widehat{D}_{3}, \widehat{D}_{4}\right) x-\frac{\Pi\left(c_{1} \widehat{D}_{1}, c_{2} \widehat{D}_{2}, c_{3} \widehat{D}_{3}, c_{4} \widehat{D}_{4}\right)}{K^{3}}\right)
$$

where $K \in\left(0, c_{4}^{2}\right)$ is given in (3.13).

Proof. Since $a_{2}, a_{4}, a_{1}-a_{3}<0$ and $K=\frac{b_{2}}{a_{2}}=\frac{b_{4}}{a_{4}}=\frac{b_{1}-b_{3}}{a_{1}-a_{3}}$, we get

$$
l_{2}(x)=a_{2} l(x), \quad l_{4}(x)=a_{4} l(x), \quad l_{1}(x)-l_{3}(x)=\left(a_{1}-a_{3}\right) l(x),
$$

where $l(x):=x-K$. Hence the polynomial in (3.5) becomes $p(x)=[l(x)]^{3} Q(x)$ where

$$
Q(x)=\left(a_{4}^{2}-a_{2}^{2}\right)^{2} l(x)+4\left(a_{1}-a_{3}\right)\left(a_{4}^{2} l_{1}(x)-a_{2}^{2} l_{3}(x)\right) .
$$

Here $Q(x)$ is definitely a linear function and from

$$
\begin{aligned}
Q^{\prime}(0) & =\left(a_{4}^{2}-a_{2}^{2}\right)^{2}+4\left(a_{1}-a_{3}\right)\left(a_{1} a_{4}^{2}-a_{3} a_{2}^{2}\right)=\Pi\left(\widehat{D}_{1}, \widehat{D}_{2}, \widehat{D}_{3}, \widehat{D}_{4}\right), \\
Q(0) & =-K\left(a_{4}^{2}-a_{2}^{2}\right)^{2}+4\left(a_{1}-a_{3}\right)\left(a_{2}^{2} b_{3}-a_{4}^{2} b_{1}\right) \\
& =-\frac{\left(b_{4}^{2}-b_{2}^{2}\right)^{2}+4\left(b_{1}-b_{3}\right)\left(b_{1} b_{4}^{2}-b_{3} b_{2}^{2}\right)}{K^{3}}=-\frac{\Pi\left(c_{1} \widehat{D}_{1}, c_{2} \widehat{D}_{2}, c_{3} \widehat{D}_{3}, c_{4} \widehat{D}_{4}\right)}{K^{3}},
\end{aligned}
$$

we conclude $Q(x)=\Pi\left(\widehat{D}_{1}, \widehat{D}_{2}, \widehat{D}_{3}, \widehat{D}_{4}\right) x-\frac{1}{K^{3}} \Pi\left(c_{1} \widehat{D}_{1}, c_{2} \widehat{D}_{2}, c_{3} \widehat{D}_{3}, c_{4} \widehat{D}_{4}\right)$.

Remark 3.14. From Theorem 3.12 we can easily prove that four of $\vec{g}_{1}, \vec{g}_{2}, \vec{g}_{3}, \vec{g}_{4}$ cannot be the solutions at the same time for a single $\tilde{c}_{66}$ : If so, $d_{1} \widehat{D}_{1}=0$ that is a contradiction.

3.5. Generic uniqueness of $\vec{g}$ for a single $\tilde{c}_{66}$. In this subsection we show that generically only one of (3.6)-(3.9) is satisfied for each root $\tilde{c}_{66} \in\left(0, c_{4}^{2}\right]$ of $p(x)$, hence generically the maximum number of possible solutions $\left(\tilde{c}_{66}, \tilde{c}_{44}, \vec{f}\right)$ is at most four.

Corollary 3.15 .

(a) Let $\tilde{c}_{66} \in\left(0, c_{4}^{2}\right]$ be a root of $p(x)$ in (3.5) and $m$ be its multiplicity. If we denote by $G\left(\tilde{c}_{66}\right)$ the number of possible $\vec{g}$ corresponding to this $\tilde{c}_{66}$, then $1 \leq G\left(\tilde{c}_{66}\right) \leq \min (m, 3)$. 
(b) The number of all possible $\left(\tilde{c}_{66}, \tilde{c}_{44}, \vec{f}\right)$ is less than or equal to the number of (multiply counted) roots of $p(x)$ in $\left(0, c_{4}^{2}\right]$, which cannot exceed four.

(c) Unless the data satisfies one of the special conditions (3.10)-(3.12), there exists only one $\vec{g}$ corresponding to a single $\tilde{c}_{66}$. So in this case, the number of all possible $\left(\tilde{c}_{66}, \tilde{c}_{44}, \vec{f}\right)$ is exactly the same as the number of (not multiply counted) roots of $p(x)$ in $\left(0, c_{4}^{2}\right]$, which cannot exceed four.

Proof. We first prove (a). For any root $\tilde{c}_{66}$, at least one of (3.6)-(3.9) is satisfied, so by Theorem 3.9 we have $G\left(\tilde{c}_{66}\right) \geq 1$. Also Remark 3.14 says that $G\left(\tilde{c}_{66}\right) \leq 3$. Hence it suffices to show $G\left(\tilde{c}_{66}\right) \leq m$ for $m=1,2$. For a simple root $(m=1)$, if $G\left(\tilde{c}_{66}\right) \geq 2$, then by Theorem 3.10 and 3.11 we get $m \geq 2$, which is a contradiction. So we should have $G\left(\tilde{c}_{66}\right) \leq 1$. For a double root $(m=2)$, if $G\left(\tilde{c}_{66}\right) \geq 3$, then by Theorem 3.12 and 3.13 we get $m \geq 3$, which is a contradiction. So we should have $G\left(\tilde{c}_{66}\right) \leq 2$. (b) is straightforward from (a), and so is (c) from Theorem 3.9 and 3.10 .

From all the above, we can summarize our algorithm as follows.

1. Make the compatible data to be well-prepared.

2. Determine possible $\tilde{c}_{66} \in\left(0, c_{4}^{2}\right]$ by finding roots of the fourth order polynomial $p(x)$ in (3.5).

3. For each $\tilde{c}_{66} \in\left(0, c_{4}^{2}\right]$ obtained above, check which one among (3.6)-(3.9) is satisfied.

4. For each case, use Theorem 3.9 to determine $\vec{g}$ (equivalently, $\tilde{c}_{44}$ and $\vec{f}$ ).

Remark 3.16. If we use the whole information of the solution $u$ of (2.2) (as opposed to only the wave front positions $\hat{T}$ ), which is actually measured in experiments, then we can apply the same arguments of Section 5 in [11]. That is, for given one of the possible triples $\left(\tilde{c}_{66}, \tilde{c}_{44}, \vec{f}\right)$ there corresponds at most one density $\rho$ corresponding to that triple under the Neumann boundary condition (for Dirichlet boundary condition, $\rho$ needs to be specified on the boundary). Therefore, in this case, we have at most four possibilities in determining four parameters $\left(\rho, c_{66}, c_{44}, \vec{f}\right)$ from the data set $\left\{u_{j}(x, t) \mid x \in \Omega, t \in(0, T)\right\}_{j=1}^{4}$.

3.5.1. Examples. Here a complete set of examples is presented showing sometimes there exist no solution, a unique solution, two solutions, three solutions, or four solutions. Here we converted the final solution into the standard coordinate system to represent $\vec{f}$.

Example 3.17. (No solution) Consider the following well-prepared data

$$
\vec{n}_{1}=(1,0,0), \quad \vec{n}_{2}=\left(-\frac{1}{\sqrt{2}},-\frac{1}{\sqrt{2}}, 0\right), \quad \vec{n}_{3}=(0,0,-1), \quad \vec{n}_{4}=\left(0, \frac{1}{2},-\frac{\sqrt{3}}{2}\right)
$$

with $c_{1}^{2}=9, c_{2}^{2}=3, c_{3}^{2}=\frac{5}{2}$ and $c_{4}^{2}=1$. Then the fourth order polynomial for $\tilde{c}_{66}$ is given by $p(x)=\frac{1}{65536}\left(18145+34592 x+21440 x^{2}+7168 x^{3}+1024 x^{4}\right)$, which has no root in $\left(0, c_{4}^{2}\right]$. Hence there exists no solution matching to the data.

Example 3.18. (Unique solution) Consider the same $\vec{n}_{j}$ as in Example 3.17 with $c_{1}^{2}=8, c_{2}^{2}=7, c_{3}^{2}=2$ and $c_{4}^{2}=1$. Then $p(x)=\frac{1}{64}\left(-188+252 x+53 x^{2}-18 x^{3}+x^{4}\right)$, which has only one root $\tilde{c}_{66}=\frac{1}{2}(9-\sqrt{137-32 \sqrt{6}}) \approx 0.6719$ in $\left(0, c_{4}^{2}\right]$. Since $\tilde{c}_{66}$ satisfies (3.7), from Theorem 3.9 we get the unique solution given by

$$
\left(\begin{array}{c}
\tilde{c}_{66} \\
\tilde{c}_{44} \\
\vec{f}
\end{array}\right) \approx\left(\begin{array}{c}
0.6719 \\
10.0514 \\
(-0.8839,-0.2777,-0.3763)
\end{array}\right)
$$


Example 3.19. (Two solutions) Consider the same $\vec{n}_{j}$ as in Example 3.17 with $c_{1}^{2}=9, c_{2}^{2}=5, c_{3}^{2}=4$ and $c_{4}^{2}=3$. Since this data satisfies none of (3.10)-(3.12), only one of $\vec{g}$ will correspond to each root of the fourth order polynomial given by

$$
p(x)=\frac{1}{4096}\left(481-1488 x+1264 x^{2}-384 x^{3}+64 x^{4}\right) .
$$

This polynomial has two roots $\xi_{1} \approx 0.5194$ and $\xi_{2} \approx 1.2439$ in $\left(0, c_{4}^{2}\right]$ where $\tilde{c}_{66}=\xi_{1}$ satisfies (3.7) and $\tilde{c}_{66}=\xi_{2}$ satisfies (3.8). So by Theorem 3.9, we get two solutions:

$$
\left(\begin{array}{c}
\tilde{c}_{66} \\
\tilde{c}_{44} \\
\vec{f}
\end{array}\right) \approx\left(\begin{array}{c}
0.5194 \\
12.4873 \\
(-0.8418,-0.0235,-0.5393)
\end{array}\right), \quad\left(\begin{array}{c}
1.2439 \\
42.2907 \\
(0.4347,-0.8625,-0.2591)
\end{array}\right) \text {. }
$$

Example 3.20. (Three solutions) Consider the same $\vec{n}_{j}$ as in Example 3.17 with $c_{1}^{2}=3, c_{2}^{2}=\frac{5}{3}, c_{3}^{2}=\frac{4}{3}$ and $c_{4}^{2}=\frac{5}{4}$. Since this data satisfies (3.12) and $\widehat{D}_{2}>0$, by Theorem $3.10(\mathrm{c})$ we get two solutions $\vec{g}_{2}$ and $\vec{g}_{3}$ for $\tilde{c}_{66}=\frac{c_{4}^{2} \widehat{D}_{4}^{2}-c_{1}^{2} \widehat{D}_{1}^{2}}{\widehat{D}_{4}^{2}-\widehat{D}_{1}^{2}}=\frac{2}{3}$. Moreover, we have $p(x)=\frac{1}{576}(3 x-2)^{2}\left[x-\left(\sqrt{2}-\frac{1}{6}\right)\right]\left[x+\left(\sqrt{2}+\frac{1}{6}\right)\right]$, which has another root $\tilde{c}_{66}=\sqrt{2}-\frac{1}{6} \approx 1.2476$ in $\left(0, c_{4}^{2}\right]$ satisfying (3.6). So we get three solutions:

$$
\begin{aligned}
\left(\begin{array}{c}
\tilde{c}_{66} \\
\tilde{c}_{44} \\
\vec{f}
\end{array}\right) \approx & \left(\begin{array}{c}
0.6667 \\
3.6795 \\
(-0.8800,0.0653,-0.4704)
\end{array}\right),\left(\begin{array}{c}
1.2476 \\
3.2525 \\
0.6667 \\
12.3205 \\
(0.4475,-0.8617,-0.2392)
\end{array}\right) .
\end{aligned}
$$

Example 3.21. (Four solutions) Consider the following well-prepared data

$$
\vec{n}_{1}=(0,0,1), \quad \vec{n}_{2}=\left(0, \frac{1}{\sqrt{2}}, \frac{1}{\sqrt{2}}\right), \quad \vec{n}_{3}=\left(-\frac{\sqrt{3}}{2}, \frac{1}{2}, 0\right), \quad \vec{n}_{4}=(-1,0,0)
$$

with $c_{1}^{2}=11, c_{2}^{2}=10, c_{3}^{2}=9.9$ and $c_{4}^{2}=9.8$. Since this data satisfies none of (3.10)-(3.12), only one of $\vec{g}$ corresponds to each root of

$$
p(x)=\frac{1}{160000}\left(14055561-6614520 x+1150900 x^{2}-88000 x^{3}+2500 x^{4}\right) .
$$

This polynomial has four roots $\xi_{1} \approx 6.2722, \xi_{2} \approx 9.4936, \xi_{3} \approx 9.6576$ and $\xi_{4} \approx 9.7766$ in $\left(0, c_{4}^{2}\right]$ where $\tilde{c}_{66}=\xi_{1}$ satisfies (3.8), $\tilde{c}_{66}=\xi_{2}$ satisfies (3.7), $\tilde{c}_{66}=\xi_{3}$ and $\tilde{c}_{66}=\xi_{4}$ satisfy (3.9). From Theorem 3.9, we get four solutions:

$$
\begin{aligned}
\left(\begin{array}{c}
\tilde{c}_{66} \\
\tilde{c}_{44} \\
\vec{f}
\end{array}\right) \approx\left(\begin{array}{c}
6.2722 \\
14.8371 \\
(-0.6418,0.1900,0.7430)
\end{array}\right), & \left(\begin{array}{c}
9.4936 \\
16.2959 \\
9.6576 \\
11.2520 \\
(0.2122,0.8564,-0.4706)
\end{array}\right), \\
\left(\begin{array}{c}
9.7766 \\
11.2149 \\
(-0.2989,0.2622,-0.9176)
\end{array}\right), & \left(\begin{array}{c}
(-0.1275,0.3649,-0.9223)
\end{array}\right) .
\end{aligned}
$$



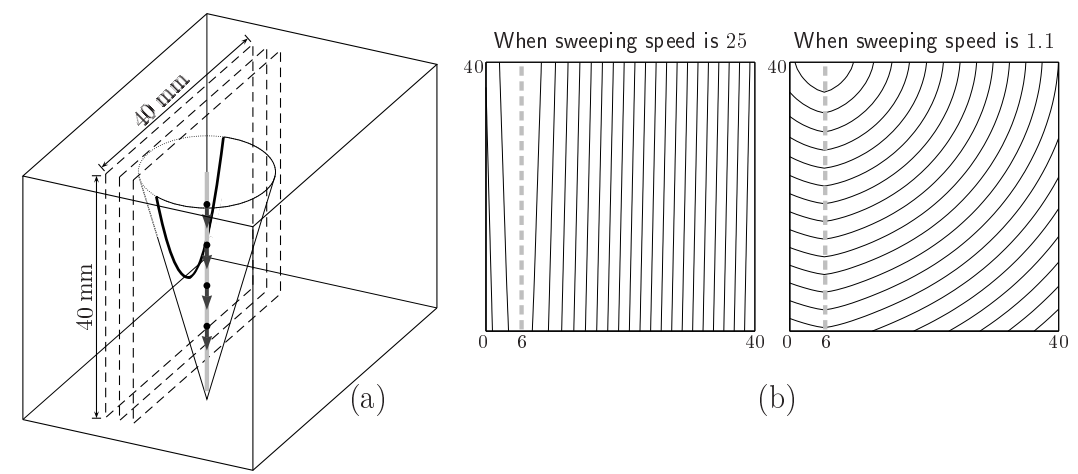

Fig. 4.1. (a) Configuration: Data are collected on three consecutive image planes (dashed lines) by either a full planar array or three parallel closely spaced linear arrays. Supersonic excitations are slightly off the imaging planes (gray line). Generated conical wave front yields parabolic intersections with each image plane. The shapes of parabola depend on the location and the sweeping speed of supersonic excitations. (b) Observed conical wave fronts on the central image plane, when the supersonic excitation line is $8 \mathrm{~mm}$ away from the central image plane and $6 \mathrm{~mm}$ apart from the left side.

4. Numerical Implementation. Here we indicate the success of the approach of using four data sets to solve the inverse problem. That is, find the triple $\left(\tilde{c}_{66}, \tilde{c}_{44}, \vec{f}\right)$ from four propagating fronts where the four normals and corresponding (estimated) wave speeds, $\left\{\left(\vec{n}_{j}, c_{j}\right)\right\}_{j=1}^{4}$ are compatible, that is, the wave speeds are all different and any three normals are linearly independent (see Definition 3.1 (a)).

Furthermore, since we develop our theory under the assumption that the medium properties may not be symmetric about the image plane we calculate the $3 \mathrm{D}$ wave front in the neighborhood of the image plane. Our supersonic excitations are assumed to be slightly out of the image plane to easily achieve the linear independence mentioned above, and we expect that this configuration could be realizable with a full planar array of transducers for 3D imaging or three lines of closely spaced transducers in a so called $2 \frac{1}{2} \mathrm{D}$ imaging setting (see Figure 4.1 (a)). For this synthetic data experiment we calculate the wave fronts using a first order anisotropic Eikonal solver based on fast marching methods with code developed at Rensselaer.

The successive supersonic imaging pushes to create the approximate line sources are made at a sweeping speed faster than the background shear wave speed and indicated by the multiple of the background shear wave speed (Mach number), hence the label supersonic, see [3]. The background wave speed is indicated in each of the labeled figures and also given in our text description below. In our examples, the pushes are either made slowly from top to bottom (1.1 sweeping speed), slowly from bottom to top ( -1.1 sweeping speed), fast from top to bottom ( 25 sweeping speed) or fast from bottom to top ( -25 sweeping speed). Each set of pushes produces a conical wave front in $3 \mathrm{D}$ whose intersection with the image plane is generally a parabola but looks like a straight line for high sweeping speeds like \pm 25 . See Figure 4.1 (b).

We show two numerical reconstructions. For the first we have uniform anisotropy where the fiber direction is out of the image plane, see Figure 4.2. The uniform anisotropic cube is $40 \mathrm{~mm}$ on a side with two excitation lines for the pushes each being $6 \mathrm{~mm}$ from the outside edge; note that the excitation lines are at different distances from the image plane with one $8 \mathrm{~mm}$ from the image plane and the other 12 $\mathrm{mm}$ from the image plane. We take separately the two sweeping speeds, \pm 1.1 , yielding 

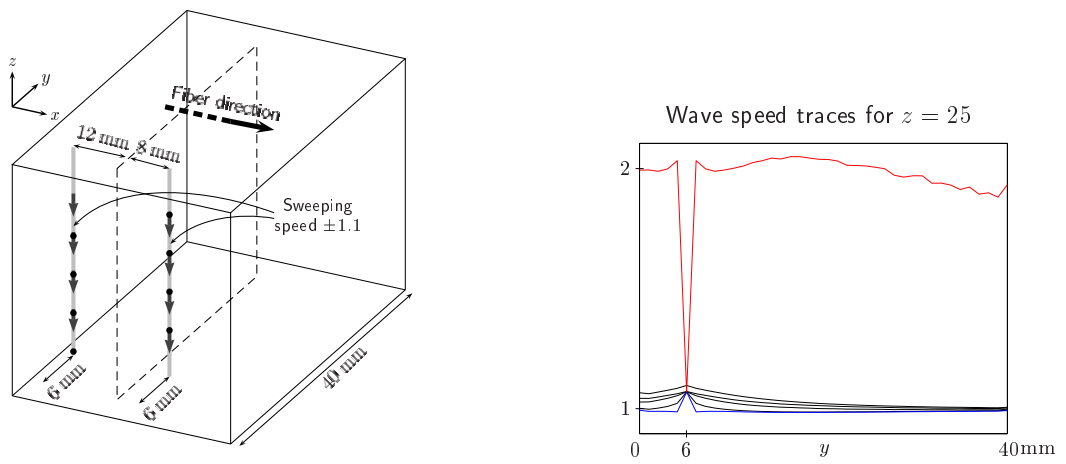

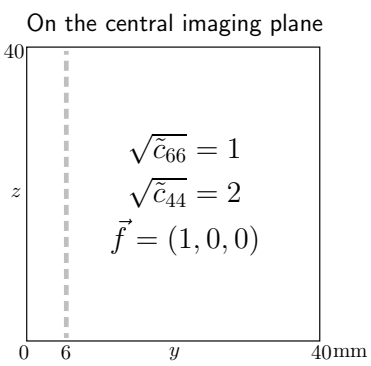

(a)

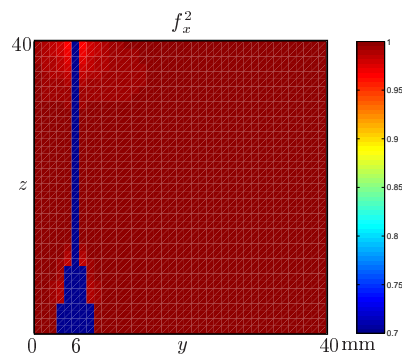

(d)

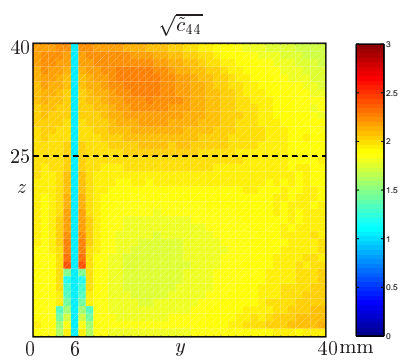

(b)

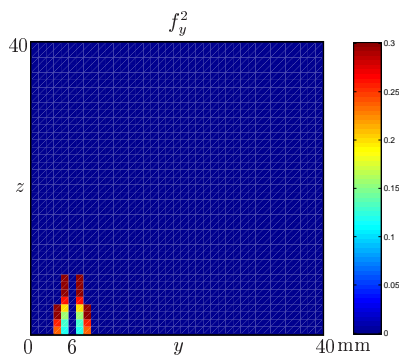

(e)

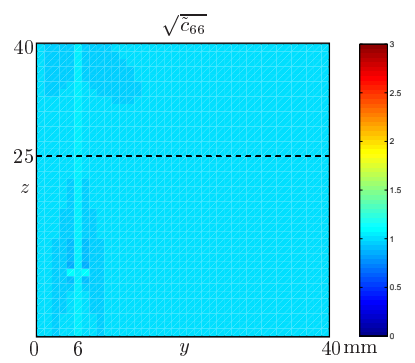

(c)

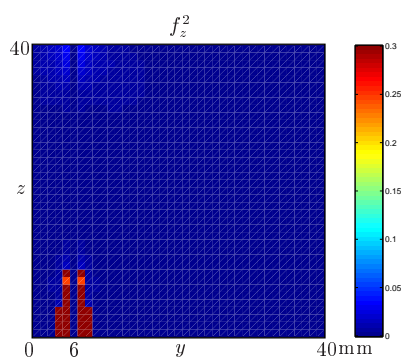

(f)

FIG. 4.2. Example 1. Top left: Orientation of fibers, central image plane (neighboring planes are omitted) and supersonic line sources. (a) Target wave speeds for uniform anisotropy, and fiber direction. Gray dashed line depicts the line source projections in the image plane. (b) Reconstructed wave speed along the fiber. (c) Reconstructed wave speed across the fiber. Top right graph shows the wave speed traces for $z=25,0<y<40$, dashed lines in (b) and (c); along the fiber (red), across the fiber (blue), estimated wave speeds $c_{j}$ in the directions $\vec{n}_{j}$ orthogonal to the four wave fronts (black). (d)-(f) Squares of the fiber direction components.

four propagating fronts. It is assumed that $\sqrt{\tilde{c}_{66}}=1, \sqrt{\tilde{c}_{44}}=2$. Setting up the three orthogonal coordinates with the $x$ coordinate out of the plane we show our results for $\sqrt{\tilde{c}_{44}}, \sqrt{\tilde{c}_{66}}$, the wave speeds along and across the fiber direction, respectively, and the squares of the fiber direction coordinates $f_{x}^{2}, f_{y}^{2}, f_{z}^{2}$. In addition we exhibit $\sqrt{\tilde{c}_{66}}$, $\sqrt{\widetilde{c}_{44}}$, and $\left\{c_{j}\right\}_{j=1}^{4}$ along the line $z=25,0<y<40$.

As we have seen in Section 3, sometimes the fourth order polynomial for $\tilde{c}_{66}$ may have multiple roots in $\left(0, c_{4}^{2}\right]$, which is the source of our non-uniqueness. In this case, we have chosen to select the largest possible root in $\left(0, c_{4}^{2}\right]$ as in all of our simulations that choice consistently gave the correct recovery. Note that there are artifacts near the projections of the excitation lines, $y=6$, onto the image plane because the fourth 

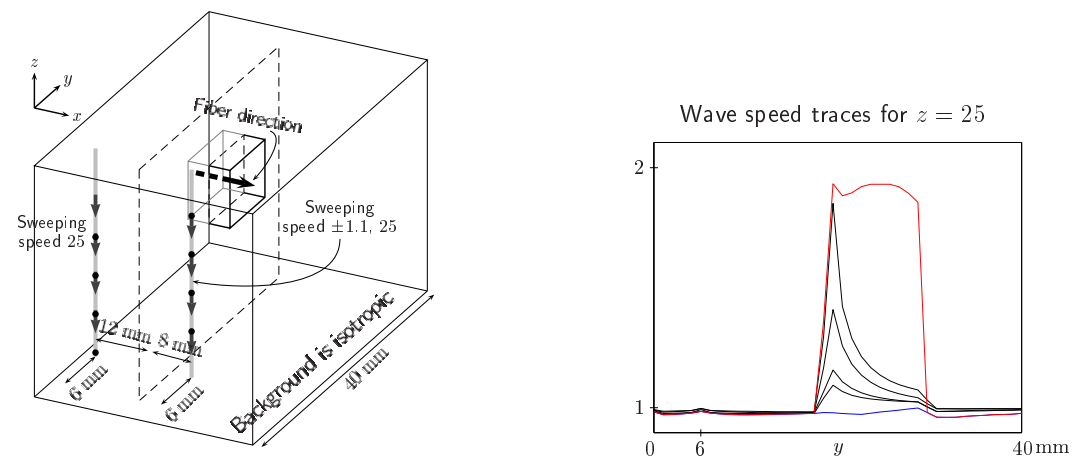
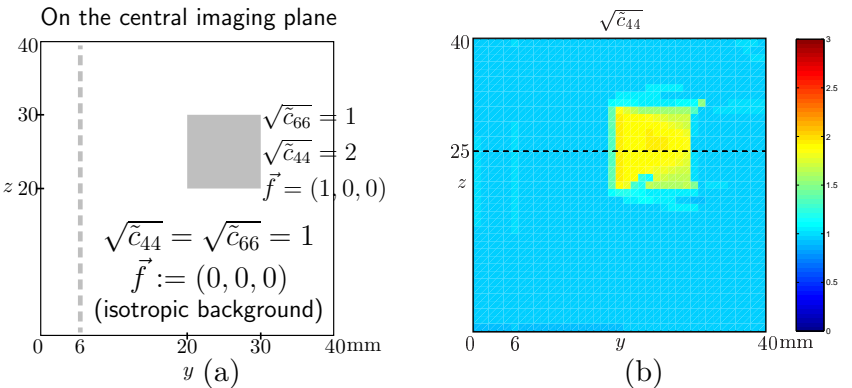

(b)

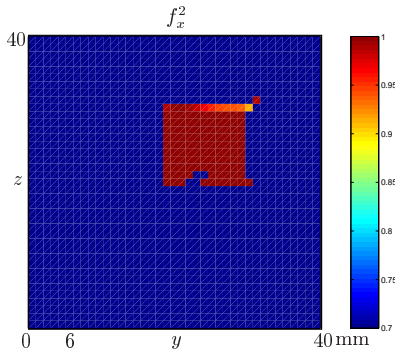

(d)

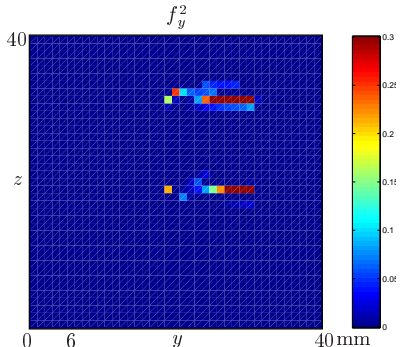

(e)

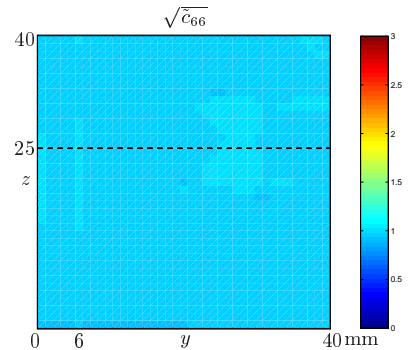

(c)

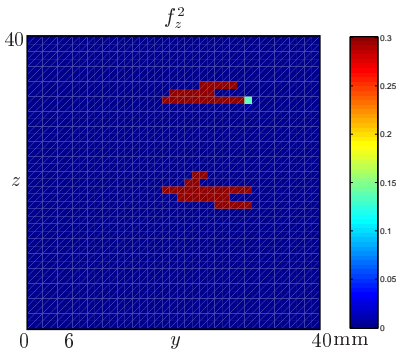

(f)

FIG. 4.3. Example 2. Top left: Orientation of fibers in the anisotropic cube inclusion and supersonic line sources. (a) Target wave speeds and fiber direction in the background and in the anisotropic cube. (b) Reconstructed wave speed along the fiber. (c) Reconstructed wave speed across the fiber. Top right graph shows the wave speed traces for $z=25,0<y<40$, dashed lines in (b) and (c); along the fiber (red), across the fiber (blue), estimated wave speeds $c_{j}$ in the directions $\vec{n}_{j}$ orthogonal to the four wave fronts (black). (d)-(f) Squares of the fiber direction components.

order polynomial is not well-defined there (there four wave normals, $\vec{n}_{j}$, are on one plane perpendicular to our image plane, i.e., $\hat{D}_{j}=0$, which yields $p(x) \equiv 0$ ). But otherwise the recovery is quite acceptable.

For our second simulation the excitation lines are in the same locations, but along one line we take the sweeping speeds, $\pm 1.1,25$, and along the second line the sweeping speed is 25 . Here the fiber is again out of the plane but only in the anisotropic cube inclusion with $10 \mathrm{~mm}$ on each side. The anisotropic inclusion is embedded in an isotropic medium, see Figure 4.3. Again the recovery is quite acceptable; note that in all images of the material properties we observe anisotropic cube edge effects, except in the image for $\sqrt{\tilde{c}_{66}}$.

Here, also in the first simulation, the points where all four wave speeds $\left\{c_{j}\right\}_{j=1}^{4}$ 
are so close (using some threshold) are considered as isotropic points. We established a threshold, $\delta=0.01$ for the first simulation and 0.04 for the second simulation, and consider the points isotropic when $\max \left\{c_{j}\right\}_{j=1}^{4}-\min \left\{c_{j}\right\}_{j=1}^{4} \leq \delta \max \left\{c_{j}\right\}_{j=1}^{4}$. For isotropic points, we assign zero vector as the fiber direction, and set $\sqrt{\tilde{c}_{44}}=\sqrt{\tilde{c}_{66}}$. As mentioned before, the fourth order polynomial is not well-defined on the excitation lines, which stems from the fact that all the four wave speeds are so close there. Because those points are considered as isotropic points, they are buried in the isotropic background in the second simulation, while in the first simulation the isotropic excitation line stands out in the anisotropic background. Compare the graphs near $y=6$ in Figure 4.2 and Figure 4.3.

5. Conclusion. Here we address the question: How do we obtain anisotropic medium properties from a set of wave fronts? Our target application is tissue shear stiffness imaging and we assume the medium is 3 dimensional. There is a fiber direction along which the wave speed, $\sqrt{\tilde{c}_{44}}$, is faster than in the plane orthogonal to the fiber where the wave speed is $\sqrt{\tilde{c}_{66}}$ and directionally independent in that plane. We show that from four wave fronts, where any three normals at each point are linearly independent, we can have up to four distinct triples $\left(\tilde{c}_{66}, \tilde{c}_{44}, \vec{f}\right)$ where $\vec{f}$ is the unit fiber direction. We exhibit examples to show multiple solutions can occur and show numerical reconstructions with synthetic data. The multiple solutions are a result of the nonlinearity in the Eikonal equation.

From our work to obtain reconstructions we have observed the importance of: (1) having well separated normals to the wave fronts and that necessitates some normals having out of image plane components; 2 the need to have multiple image planes to capture all three components of the normals; and (3) that in a high contrast subregion embedded in a constant medium, initially well separated normals may align themselves (the angle between their normals becomes smaller) at some points and at other points the angle may become greater. This angle change may occur also at points beyond that subregion. The degree of this angle change depends on the wave speed contrast, size of inclusion and the initial incident directions. This indicates important features in experimental design when wave fronts are used to image anisotropic properties of the kind modeled in this paper.

Acknowledgments. We have benefitted from discussions with Antoinette Maniatty, Maarten de Hoop, William Symes, Lizabeth Rachele and Gunther Uhlmann. Funding for the work of Yoon was provided by the NSF Focus Group Grant DMS 0101458 with partial support from IPAM during Fall 2003, IMA during Fall 2005, and his start-up grant from Clemson University. McLaughlin was partially supported by NSF Focus Group Grant DMS 0101458, ONR Grant N00014-96-1-0349, ONR Grant N00014-05-1-0600, NIBIB Grant 1R21EB003000-01, and IPAM during Fall 2003. The work of Renzi was partially supported by NSF VIGRE grant No DMS 9983646 and IPAM during Fall 2003.

\section{REFERENCES}

[1] Bercoff J, Chaffai S, Tanter M, Sandrin L, Catheline S, Fink M, Gennisson J-L, and Meunier M 2003 In vivo breast tumor detection using transient elastography Ultrasound in Med. and Biol. 29: 1387-96.

[2] Bercoff J, Tanter M, Chaffai S, and Fink M 2002 Ultrafast imaging of beamformed shear waves induced by the acoustic radiation force: application to transient elastography Proceedings of the 2002 IEEE Ultrasonics Symposium 2: 1899-1902. 
[3] Bercoff J, Tanter M, and Fink M 2004 Supersonic shear imaging: a new technique for soft tissue elasticity mapping IEEE Transactions on Ultrasonics, Ferroelectrics, and Frequency Control 51: 396-409.

[4] Doyley M M, Meaney P M, and Bamber J C 2000 Evaluation of an iterative reconstruction method for quantitative elasticity Phys. Med. Biol. 45: 1521-40.

[5] Eller M, Isakov V, Nakamura G, and Tataru D 2000 Uniqueness and stability in the Cauchy problem for Maxwell and elasticity systems in Nonlinear Partial Differential Equations College de France Seminar 14 (Chapman and Hall/CRC Press)

[6] Evans L C 1998 Partial Differential Equations American Mathematical Society (GSM/19)

[7] Fatemi F and Greenleaf J F 1998 Ultrasound-simulated vibro-acoustic spectography, Science 280: 82-85.

[8] Gao L, Parker K J, and Alam S K 1995 Sonoelasticity imaging: theory and experimental verification J. Acoust. Soc. Am. 97: 3875-80.

[9] Gennisson J-L, Catheline S, Chaffai S, and Fink M 2003 Transient elastography in anisotropic medium: Application to the measurement of slow and fast shear wave speeds in muscles $J$. Acoust. Soc. Am. 114: 536-41.

[10] Ji L, McLaughlin J R, Renzi D and Yoon J R 2003 Interior elastodynamics inverse problems: shear wave speed reconstruction in transient elastography Inverse Problems 19: S1-S29.

[11] McLaughlin J R and Yoon J R 2004 Unique identifiability of elastic parameters from time dependent interior displacement measurement Inverse Problems 20: 25-45.

[12] McLaughlin J and Renzi D 2005 Shear wave speed recovery in transient elastography and supersonic imaging using propagating fronts Submitted for publication.

[13] McLaughlin J, Renzi D 2005 Using level set based inversion of arrival times to recover shear wave speed in transient elastography and supersonic imaging Submitted for publication.

[14] McLaughlin J, Parker K, Renzi D, and Wu Z 2005 Shear wave speed recovery using moving interference patterns obtained in sonoelastography experiments Submitted for publication.

[15] Muthpillai R, Lomas D J, Rossman P J, Greenleaf J F, Manduca A, and Ehman R I 1995 Magnetic resonance elastography by direct visualization of propagating acoustic strain waves Science 269: 1854-1857.

[16] Nightingale K R, Palmeri J L , Nightingale R W, and Trahey G E 2001 On the feasibility of remote palpation using acoustic radiation force J. Acoust. Soc. Am. 110: 625-634.

[17] Ophir J, Cespedes I, Ponnekanti H, Yazdi Y, and Li X 1991 Elastography: a quantitative method for imaging the elasticity of biological tissues Ultrason. Imaging 13: 111-34.

[18] Sinkus R, Lorenzen J, Schrader D, Lorenzen M, Dargatz M, and Holz D 2000 High-resolution tensor MR elastography for breast tumor detection Phys. Med. Biol. 4: 1649-1664.

[19] Sinkus R, Tanter M, Catheline S, Lorenzen J, Kuhl C, Sondermann E, and Fink M 2005 Imaging anisotropic and viscous properties of breast tissue by magnetic resonance-elastography Magnetic Resonance in Medicine 53: 372-387.

[20] Taylor L S, Porter B C, Rubens D J, and Parker K J 2000 Three-dimensional sonoelastography: principles and practices Phys. Med. Biol. 45: 1477-1494.

[21] Weaver J, Doyley M, Van Houten E, Hood M, Qin X C, Kennedy F, Poplack S, and Paulsen K 2002 Evidence of the anisotropic nature of the mechanical properties of breast tissue Med. Phys. 29: 1291-1291.

[22] Wu Z, Taylor L S, Rubens D J, and Parker K J 2004 Sonoelastographic imaging of interference patterns for estimation of the shear velocity of homogeneous biomaterials Phys. Med. Biol. 49: 911-922. 